\title{
FREE PRODUCTS OF INVERSE SEMIGROUPS
}

BY

PETER R. JONES ${ }^{1}$

\begin{abstract}
A structure theorem is provided for the free product $S$ inv $T$ of inverse semigroups $S$ and $T$. Each element of $S$ inv $T$ is uniquely expressible in the form $\varepsilon(A) a$, where $A$ is a certain finite set of "left reduced" words and either $a=1$ or $a=a_{1} \cdots a_{m}$ is a "reduced" word with $a a_{m}^{-1} \in A$. (The word $a_{1} \cdots a_{m}$ in $S \operatorname{sgp} T$ is called reduced if no letter is idempotent, and left reduced if exactly $a_{m}$ is idempotent; the notation $\varepsilon(A)$ stands for $\Pi\left\{a a^{-1}: a \in A\right\}$.) Under a product remarkably similar to Scheiblich's product for free inverse semigroups, the corresponding pairs $(A, a)$ form an inverse semigroup isomorphic with $S$ inv $T$.

This description enables various properties of $S \operatorname{inv} T$ to be determined. For example $(S \operatorname{inv} T) \backslash(S \cup T)$ is always completely semisimple and each of its subgroups is isomorphic with a finite subgroup of $S$ or $T$. If neither $S$ nor $T$ has a zero then $S$ inv $T$ is fundamental, but in general fundamentality itself is not preserved.
\end{abstract}

1. Introduction. From purely universal algebraic considerations (see, for example, $[1, \S 72]$ ) it follows that since inverse semigroups form a variety, the free product (or "coproduct") of any family of inverse semigroups exists and, moreover, is generated by isomorphic copies of the members of that family. However, the first attempt to actually describe the free product $S$ inv $T$ of two inverse semigroups was made in 1973 by Preston [11] as a certain quotient of a free semigroup product. This followed the first concrete and useful description of free inverse semigroups, given by Scheiblich [12]. Preston's description of $S$ inv $T$ gave no insight into its structure, however, and he suggested the possibility of a concrete structure theorem along the lines of Scheiblich's. Various special cases have since been covered: when both $S$ and $T$ are groups, $S$ inv $T$ was described by Knox [8] and McAlister [9]; when both $S$ and $T$ are E-unitary, a description of $S$ inv $T$ using graphs was obtained by the author [7].

In this paper a structure theorem for the free product of any two inverse semigroups (which extends easily to arbitrary families) $S$ and $\mathrm{T}$ is provided. This enables a canonical form for its elements to be provided, Green's relations to be determined and various structural and preservational properties to be discussed. In view of the complexity of some of the intermediate results it is not surprising that the inverse semigroup constructed is somewhat complicated to describe. (Its applications, though, should be proof of its practicality.) The construction is in several stages, which are summarized in the remainder of this section.

Received by the editors February 24, 1983. The results were first presented at a meeting of the American Mathematical Society, East Lansing, Michigan, on November 13, 1982.

1980 Mathematics Subject Classification. Primary 20M05.

Key words and phrases. Inverse semigroup, free product, canonical form, structural and preservational properties.

${ }^{1}$ This research was supported by NSF grant MCS-8201023. 
In $\$ 3$ a (finite) process is given by which any element $x$ of $S \operatorname{inv} T$ may be expressed in the form $\varepsilon(A) a$, where $A$ belongs to a certain family $\mathcal{Y}$ of "canonical sets" (each consisting of a finite number of words in $S \operatorname{sgp} T$ ),

$$
\varepsilon(A)=\prod\left\{a a^{-1}: a \in A\right\},
$$

and $a$ is an "associate" of $A$. More precisely, a finite nonempty subset $A$ of $S \operatorname{sgp} T$ is canonical if (i) each word $a_{1} \cdots a_{m}$ in $A$ is left reduced (that is, $a_{m}$ is its unique idempotent letter), (ii) $A$ is prefix closed (if $a_{1} \cdots a_{m} \in A$ then $a_{1} \cdots a_{i} a_{i}^{-1} \in A$, $1 \leqslant i \leqslant m$ ), and (iii) $A$ has unique last letters (if $a_{1} \cdots a_{m-1} a_{m} \in A$ and $a_{1}$ $\cdots a_{m-1} b_{m} \in A$ then $\left.a_{m}=b_{m}\right) ; a$ is an associate of $A$ if either $a=1$, the empty word, or $a=a_{1} \cdots a_{m}$ is reduced (none of its letters is idempotent) and $a a_{m}^{-1} \in A$. If $x$ is idempotent then $a=1$.

In $\S 4$ representations of $S$ and $T$ in $\mathscr{G}_{0,}$, the symmetric inverse semigroup on the set $\mathcal{Y}$, are defined and extended to a representation $\rho$ of $S \operatorname{inv} T$ in $\mathscr{q}_{\mathscr{Y}}$. This representation is then used to define a partial order $\leqslant$ on $\mathscr{Y}$ in such a way that $(S \operatorname{inv} T) \rho$ consists of partial order isomorphisms of the poset $(\mathcal{Y}, \leqslant)$. The sequence of maps

$$
A \rightarrow \varepsilon(A) \rightarrow \rho_{\varepsilon(A)} \rightarrow \Delta \rho_{\varepsilon(A)}=\mathscr{\mathcal { Y }}_{A} \rightarrow A
$$

(where $\Delta \rho_{\varepsilon(A)}$ denotes the domain of the partial transformation $\rho_{\varepsilon(A)}$ and $\mathscr{Y}_{A}$ is the principal ideal of $\mathscr{Y}$ generated by $A$ ) is shown to be an isomorphism, so that $\mathcal{Y}$ is a semilattice, $\mathcal{O} \cong E(S$ inv $T)$ and $\rho$ is an idempotent separating representation of $S$ inv $T$ in the Munn semigroup $T_{\mathscr{Y}}$ (which consists of all isomorphisms between principal ideals of $\mathcal{Y}$ ). In fact $\rho$ is essentially the Munn representation.

In $\$ 5$ a product is defined on the set $F$ of all pairs $(A, a)$, where $A \in \mathcal{O}$ and $a$ is an associate of $A$. Using a representation $\tau$ of $S \operatorname{inv} T$ in $\oint_{F}$ it is similarly shown that $F$ is an inverse semigroup, $F \cong S$ inv $T$ and $\tau$ is faithful. (Essentially $\tau$ is just the Preston-Vagner representation.) This shows that the "canonical form" given in $\S 3$ is unique.

In the final two sections Green's relations are found on $F$ and various structural (§5) and preservational $(\S 6)$ properties are discussed.

2. Preliminaries. If $S$ and $T$ are inverse semigroups then $S \operatorname{sgp} T$ and $S \operatorname{inv} T$ will denote their free product in the categories of semigroups and of inverse semigroups, respectively, defined by the usual universal properties. The elements of $S \operatorname{sgp} T$ are words $a=a_{1} \cdots a_{m}$ over $S \cup T$ no two of whose adjacent letters belong to the same factor $S$ or $T$. In a natural way these words may be thought of as elements of $S$ inv $T$, and since $S$ and $T$ may be considered as generating $S \operatorname{inv} T$, every element of $S$ inv $T$ may be expressed in this way, though not uniquely.

To avoid repetition the expression " $a \in S \operatorname{sgp} T$ " will assume $a$ has the form $a_{1} \cdots a_{m}$ (the $a_{i}$ 's alternately from $S$ and $T$ ), where $m=l(a)$, the length of $a$.

Such a word $a$ will be termed (i) reduced if no $a_{i}$ is idempotent, (ii) right idempotent if $a_{m}$ is idempotent and (iii) left reduced if $a_{m}$, but no other letter, is idempotent. The empty word, denoted 1 , will be assumed to be reduced, of length 0 , but not right idempotent. 
The right idempotent word $a$ will be called above the right idempotent word $b$ if $l(b)=l(a)$ and, for each $i, a_{i} \geqslant b_{i}$ in the natural partial order on the appropriate factor.

An internal idempotent of a right idempotent word $a$ is any idempotent letter $a_{r}$ with $r<m$; in that event we denote by $\hat{a}_{r}$ the right idempotent word obtained from $a$ after deleting $a_{r}$. Thus $\hat{a}_{1}=a_{2} \cdots a_{m}, \hat{a}_{m-1}=a_{1} \cdots\left(a_{m-2} a_{m} a_{m-2}^{-1}\right)$ (when $\left.m>2\right)$, and $\hat{a}_{r}=a_{1} \cdots\left(a_{r-1} a_{r+1}\right) \cdots a_{m}$ otherwise.

For each $a \in S \operatorname{sgp} T$ let

$$
\operatorname{pre}(a)=\left\{a_{1} a_{1}^{-1}, a_{1} a_{2} a_{2}^{-1}, \ldots, a_{1} \cdots a_{m-1} a_{m-1}^{-1}, a_{1} \cdots a_{m} a_{m}^{-1}\right\},
$$

the set of prefixes of $a$. A subset $A$ of $S \operatorname{sgp} T$ will be called prefix closed if $\operatorname{pre}(a) \subseteq A$ for all $a \in A$.

Let $\mathbb{Q}$ (or $\mathcal{Q}(S, T)$ when the possibility of confusion arises) denote the collection of all finite, nonempty, prefix closed sets of right idempotent words. We shall call these precanonical sets. If $A \in \mathbb{Q}$ denote by $A_{S}$ and $A_{T}$ the sets of words in $A$ whose first letter belongs to $S$ or $T$, respectively. The weight of $A$ is defined by $w(A)=\Sigma_{a \in A} l(a)$.

With any right idempotent word $a$ may be associated the idempotent $\varepsilon(a)=a a^{-1}$ in $S$ inv $T$; if $A \in \mathbb{Q}$ put $\varepsilon(A)=\prod_{a \in A} \varepsilon(a)$. Note that if $a$ and $b$ are right idempotent and $a$ is above a prefix of $b$, then $\varepsilon(a) \geqslant \varepsilon(b)$, for

$$
\begin{aligned}
&\left(a a^{-1}\right)\left(b b^{-1}\right)=a_{1}\left(a_{2} \cdots a_{m} \cdots a_{2}^{-1}\right)\left(a_{1}^{-1} b_{1}\right)\left(b_{2} \cdots b_{n} \cdots b_{2}^{-1}\right) b_{1}^{-1} \\
&=a_{1}\left(a_{1}^{-1} b_{1}\right)\left(a_{2} \cdots a_{m} \cdots a_{2}^{-1}\right)\left(b_{2} \cdots b_{n} \cdots b_{2}^{-1}\right) b_{1}^{-1}, \\
& \text { commuting idempotents in } S \text { inv } T \\
&= b_{1}\left(a_{2} \cdots a_{m} \cdots a_{2}^{-1}\right)\left(b_{2} \cdots b_{n} \cdots b_{2}^{-1}\right) b_{1}^{-1}, \\
& \text { since } a_{1} \geqslant b_{1}, \text { so } a_{1}^{-1} b_{1}=b_{1}^{-1} b_{1} .
\end{aligned}
$$

By induction this yields

$$
b_{1} \cdots b_{m-1}\left(a_{m}\right)\left(b_{m} \cdots b_{n} \cdots b_{m}^{-1}\right) b_{m-1}^{-1} \cdots b_{1}^{-1}=b b^{-1} \text {, since } a_{m} \geqslant b_{m} b_{m}^{-1} \text {. }
$$

In particular, for any right idempotent word $a$,

$$
\varepsilon(a)=\varepsilon(\operatorname{pre}(a)) .
$$

Finally, if $A \in \mathbb{Q}$ the word $a$ in $(S \operatorname{sgp} T)^{1}$ is an associate of $A$ if either $a=1$ or $a_{m}$ is nonidempotent and $a a_{m}^{-1} \in A$.

The terminology and notation will, in general, be that of Howie [3]. In particular we refer the reader to Chapter 5 for the definitions and elementary properties of the natural partial order on an inverse semigroup, the symmetric inverse semigroup $q_{X}$ on a set $X$ and the Munn semigroup $T_{E}$ on a semilattice $E$.

We deviate from the notation in [3] by denoting by $\Delta \phi$ and $\nabla \phi$ the domain and range, respectively, of a member $\phi$ of $\Phi_{X}$. In addition, the semilattice of idempotents of an inverse semigroup $U$ will be denoted $E(U)$.

3. A reduction algorithm. In this section a procedure is described which reduces any element of $S \operatorname{inv} T$ to a "canonical form", the uniqueness of which will be proved in $\S \S 4,5$. Some further definitions are first required. 
Let $A$ be a precanonical set (that is, $A \in \mathcal{Q}$ ): then $A$ is called left reduced if it consists of left reduced words; $A_{S}\left[A_{T}\right]$ has unique last letters if for any $a=a_{1} \cdots a_{m}$ and $b=b_{1} \cdots b_{m}$ in $A_{S}\left[A_{T}\right], a_{i}=b_{i}$ for all $i<m$ implies $a_{m}=b_{m} ; A$ itself has unique last letters if both $A_{S}$ and $A_{T}$ do. Observe that if $a_{1} \cdots a_{m}$ and $b_{1} \cdots b_{n}$ are any two words in $A_{S}$ which agree on their first $k-1$ letters, then by prefix closure both $a_{1} \cdots a_{k-1}\left(a_{k} a_{k}^{-1}\right)$ and $b_{1} \cdots b_{k-1}\left(b_{k} b_{k}^{-1}\right)$ belong to $A_{S}$; thus $A_{S}$ has unique last letters if and only if any two words which agree on their first $k-1$ letters have GR-related $k$ th letters. Observe, also, that in a set $A$ with unique last letters both $A_{S}$ and $A_{T}$ have at most one word of length one. (By prefix closure either $A_{S}$ or $A_{T}$ has such a word, which is an idempotent.)

A precanonical set $A$ is called canonical if it is both left reduced and has unique last letters; $\mathcal{O}$ will denote the family of all canonical sets. Observe that any associate of a canonical set is necessarily reduced. Note also that for any left reduced word $a$, $\operatorname{pre}(a)$ is a canonical set.

The remainder of the section is devoted to showing that each element of $S$ inv $T$ is expressible in the form $\varepsilon(A) a$, where $A \in \mathcal{Y}$ and $a$ is an associate of $A$. To begin, let $x=x_{1} \cdots x_{n} \in S \operatorname{sgp} T$ and note that

$$
x x^{-1}=\varepsilon\left(x_{1} \cdots x_{n} x_{n}^{-1}\right)=\varepsilon\left(\operatorname{pre}\left(x_{1} \cdots x_{n} x_{n}^{-1}\right)\right),
$$

where $\operatorname{pre}\left(x_{1} \cdots x_{n} x_{n}^{-1}\right) \in \mathcal{Y}$. Let $x^{\prime}$ be the word obtained from $x$ by "deleting terminal idempotents": that is, $x^{\prime}=1$ if each letter of $x$ is idempotent, or $x^{\prime}=x_{1}$ $\cdots x_{i}$ if $x_{i}$ is the last nonidempotent letter. An induction establishes that $x=$ $\varepsilon\left(\operatorname{pre}\left(x_{1} \cdots x_{n} x_{n}^{-1}\right)\right) x^{\prime}$ and $x^{\prime}$ is clearly an associate of $\operatorname{pre}\left(x_{1} \cdots x_{n} x_{n}^{-1}\right)$. Thus to obtain the required expression it is sufficient to show that for any precanonical set $A$ and associate $a$ of $A$ there is a canonical set $Y$ and associate $y$ of $Y$ such that $\varepsilon(A) a=\varepsilon(Y) y$.

Let $A \in \mathbb{Q}$. Reduction of $A$ to a set in $\mathscr{P}$ is treated first. This consists of a repetition of two basic steps.

1. Idempotent deletion. If $A$ is left reduced, put $L(A)=A$; if not, let $i$ be the least positive integer for which the $i$ th letter of some word $a$ in $A$ is an internal idempotent: let $L(A)$ be the set obtained from $A$ by replacing each such word $a$ by $\hat{a}_{i}$.

2. Last letter reduction. If $A$ has unique last letters put $R(A)=A$; if not, there is a least positive integer $k$ for which, for some $x$ and $y$ of length $k$ in $A_{S}$, or in $A_{T}$, $x_{j}=y_{j}$ for all $j<k$ but $x_{k} \neq y_{k}$. Then let $R(A)$ be the set obtained from $A$ by replacing each $a$ of length $m \geqslant k$ by $a_{1} \cdots\left(e_{k} a_{k}\right) \cdots a_{m}$, where $e_{k}=e_{k}(a)=$ $\Pi\left\{f: a_{1} \cdots a_{k-1} f \in A_{S}\left[A_{T}\right]\right\}$. (Thus only words of length $k$ or more are modified.)

Lemma 3.1. Let $A \in \mathbb{Q}$. Then (i) $L(A) \in \mathbb{Q}, \varepsilon(L(A))=\varepsilon(A)$ and, if $L(A) \neq A$, $w(L(A))<w(A)$; and (ii) $R(A) \in Q, \varepsilon(R(A))=\varepsilon(A)$ and, if $R(A) \neq A, w(R(A))$ $<w(A)$.

Proof. (i) Assume $L(A) \neq A$ and let $i$ be as in the definition. Clearly $L(A)$ consists of right idempotent words, so it remains to show it is prefix closed. Let $b \in L(A)$. If $b \in A$ and $b_{i}$ is not an internal idempotent then $b$ is unchanged by the 
formation of $L(A)$, so $\operatorname{pre}(b) \subseteq L(A)$, since the same is true of each of its prefixes. Otherwise $b=\hat{a}_{i}$ for some $a \in A$ with internal idempotent $a_{i}$. Let $c$ be a prefix of $b$ : if $l(c)<i-1$ then $c \in A$ and is unchanged by the formation of $L(A)$, so $c \in L(A)$; on the other hand if $l(c) \geqslant i-1$, then $c$ is obtained from some prefix of $a$, of length $i+1$ or more, and so belongs to $L(A)$. Thus pre $(b) \subseteq L(A)$.

To show $\varepsilon(L(A))=L(A)$ it is sufficient to show $\varepsilon\left(a_{1} \cdots a_{i}\right) \varepsilon\left(\hat{a}_{i}\right)=\varepsilon(a)$ for each $a$ in $A$ for which $a_{i}$ is an internal idempotent (since in that case $a_{1} \cdots a_{i}$ belongs to both $A$ and $L(A)$ ). For $i=1$ this is evident. Otherwise, by commuting idempotents in $S \operatorname{inv} T$,

$$
\begin{aligned}
\varepsilon\left(a_{1} \cdots a_{i}\right) \varepsilon\left(\hat{a}_{i}\right)= & \left(a_{1} \cdots a_{i-1} a_{i} a_{i-1}^{-1} \cdots a_{1}^{-1}\right) \\
& \cdot\left(a_{1} \cdots\left(a_{i-1} a_{i+1}\right) \cdots a_{m} \cdots\left(a_{i+1}^{-1} a_{i-1}^{-1}\right) \cdots a_{1}^{-1}\right) \\
= & \left(a_{1} \cdots a_{i-1}\right)\left(a_{i-1}^{-1} \cdots a_{1}^{-1}\right)\left(a_{1} \cdots a_{i-1}\right) \\
& \cdot a_{i}\left(a_{i+1} \cdots a_{m} \cdots a_{i+1}^{-1}\right) a_{i}^{-1}\left(a_{i-1}^{-1} \cdots a_{1}^{-1}\right) \\
= & \varepsilon(a) .
\end{aligned}
$$

Finally, for each such $a, l\left(\hat{a}_{i}\right)<l(a)$, so $w(L(A))<w(A)$.

(ii) Assume $R(A) \neq A$ and let $k$ be as in the definition. It is clear that $R(A) \in \mathbb{Q}$. Let $b=b_{1} \cdots b_{k} \in A_{S}$, say, and let $X_{b}=\left\{a \in A: b_{1} \cdots b_{k-1}=a_{1} \cdots a_{k-1}\right\}$. Then

$$
\begin{aligned}
\varepsilon\left(X_{b}\right) & =\prod_{a \in X_{b}} \varepsilon(a) \\
& =\prod_{a \in X_{b}} b_{1} \cdots b_{k-1} a_{k} \cdots a_{m} \cdots a_{k}^{-1} b_{k-1}^{-1} \cdots b_{1}^{-1} \\
& =b_{1} \cdots b_{k-1} \cdot \prod_{a \in X_{b}}\left(a_{k} \cdots a_{m} \cdots a_{k}^{-1}\right) \cdot b_{k-1}^{-1} \cdots b_{1}^{-1} \\
& =b_{1} \cdots b_{k-1} \cdot \prod_{a \in X_{b}} a_{k} a_{k}^{-1} \cdot \prod_{a \in X_{b}}\left(a_{k} \cdots a_{m} \cdots a_{k}^{-1}\right) \cdot b_{k-1}^{-1} \cdots b_{1}^{-1} \\
& =b_{1} \cdots b_{k-1} e_{k} \cdot \prod_{a \in X_{b}}\left(a_{k} \cdots a_{m} \cdots a_{k}^{-1}\right) \cdot b_{k-1}^{-1} \cdots b_{1}^{-1} \\
& =b_{1} \cdots b_{k-1} \prod_{a \in X_{b}}\left(\left(e_{k} a_{k}\right) \cdots a_{m} \cdots\left(e_{k} a_{k}\right)^{-1}\right) \cdot b_{k-1}^{-1} \cdots b_{1}^{-1} \\
& =\varepsilon\left(Y_{b}\right),
\end{aligned}
$$

where $Y_{b}$ is the subset of $R(A)$ obtained from $X_{b}$.

Since $A$ is the union of such sets $X_{b}$ where $l(b)=k$, together with the set of words of length less than $k$ (which are unchanged), $\varepsilon(R(A))=\varepsilon(A)$.

Finally, since words of $A$ are replaced by words in $R(A)$ of equal length, to show $w(R(A))<w(A)$ it is sufficient to show $|R(A)|<|A|$. Now by assumption there exist $a=a_{1} \cdots a_{k}$ and $b=a_{1} \cdots a_{k-1} b_{k}$ in $A_{S}$ (without loss of generality), with $a_{k} \neq b_{k}$, and $a$ is replaced by $a_{1} \cdots a_{k-1}\left(e_{k} a_{k}\right), b$ by $a_{1} \cdots a_{k-1}\left(e_{k} b_{k}\right)$, where $e_{k}=\prod\left\{f: a_{1} \cdots a_{k-1} f \in A_{s}\right\}$. But $a_{k}$ and $b_{k}$ are idempotent, so $a_{k}, b_{k} \geqslant e_{k}$ and therefore $e_{k} a_{k}=e_{k}=e_{k} b_{k}$. Thus $R(A)$ contains at least one less word than $A$. 
Corollary 3.2. For any precanonical set $A$ the sequence $A, L(A), R L(A)$, $L R L(A), \ldots$ terminates in a canonical set $\operatorname{cl}(A)$ and $\varepsilon(\operatorname{cl}(A))=\varepsilon(A)$.

Proof. Since at each nontrivial step in the sequence the weight is reduced, the sequence must terminate, and $\operatorname{cl}(A)$ clearly is left reduced and has unique right ends.

Again let $A \in \mathbb{Q}$ and suppose $a$ is an associate of $A$. If $a=1$ put $L(a)=R(a)=1$. Otherwise $a_{m}$ is nonidempotent and $a a_{m}^{-1} \in A$. Each reduction step applied to $A$ now corresponds to a reduction of $a$.

1. Idempotent deletion. If $A=L(A)$ put $L(a)=a$; otherwise let $i$ be as in the definition of $L(A)$. If $a_{i}$ is idempotent, $i<m$, and

(a) $i=1$, put $L(a)=a_{2} \cdots a_{m}$;

(b) $i>1$ and, if $i=m-1, a_{m-2} a_{m}$ is nonidempotent, put $L(a)=a_{1}$ $\cdots\left(a_{i-1} a_{i+1}\right) \cdots a_{m}$;

(c) $i=m-1$ and $a_{m-2} a_{m}$ is idempotent, put $L(a)=a_{1} \cdots a_{m-3}(=1$ if $i=2)$;

Otherwise (that is, when $a_{i}$ is not idempotent, or when $m \leqslant i$ ) put $L(a)=a$.

2. Last letter reduction. If $R(A)=A$ put $R(a)=a$; if not, let $k$ be as in the definition of $R(A)$. If $m<k$ put $R(a)=a$. If $m \geqslant k$ let $R(a)$ be the word obtained from $a_{1} \cdots\left(e_{k} a_{k}\right) \cdots a_{m}$ by deleting terminal idempotents. (Here $e_{k}=$ $e_{k}\left(a_{1} \cdots a_{k} a_{k}^{-1}\right)$.)

Lemma 3.2. Let $A \in \mathbb{Q}$ and let $a$ be an associate of $A$. Then (i) $L(a)$ is an associate of $L(A)$ and $\varepsilon(A) a=\varepsilon(L(A)) L(a)$, and (ii) $R(a)$ is an associate of $R(A)$ and $\varepsilon(A) a=\varepsilon(R(A)) R(a)$.

Proof. If $a=1$ this is obvious from Lemma 3.1. Now assume $a \neq 1$.

(i) If $L(a)=a$ this is again obvious for then $a a_{m}^{-1} \in L(A)$. Otherwise $a_{i}$ is idempotent and $i<m$. In cases (a) and (b) $L(a)$ is an associate of $L(A)$, since this set contains $\left(\widehat{a a}_{m}^{-1}\right)_{i}$. In case (c) either $L(a)=1$ or $i>2$ and $L(a)=a_{1} \cdots a_{m-3}$ is an associate of $L(A)$. (Note that $a_{1} \cdots a_{m} a_{m}^{-1} \in A$, so $a_{j}$ is nonidempotent for $j<i=m-1$.) Thus $a_{1} \cdots a_{m-3} a_{m-3}^{-1} \in L(A)$ and $a_{m-3}$ is nonidempotent. Using arguments similar to that of the last paragraph of the proof of Lemma 3.1(i), it is routine to verify that $\varepsilon(L(A)) L(a)=\varepsilon(L(A)) a(=\varepsilon(A) a)$ in each case.

(ii) If $R(a)=a$ this is once more obvious. If $m \geqslant k$ then since $a_{1} \cdots\left(e_{k} a_{k}\right)$ $\cdots a_{m} a_{m}^{-1}$ (or $a_{1} \cdots\left(e_{k} a_{k}\right)\left(e_{k} a_{k}\right)^{-1}$, if $m=k$ ) belongs to $R(A)$, it is easily verified that $R(a)$ is an associate of $R(A)$. Now observe that (for $m \geqslant k$ )

$$
\begin{gathered}
\varepsilon\left(a_{1} \cdots\left(e_{k} a_{k}\right) \cdots a_{m} a_{m}^{-1}\right) a \\
=\varepsilon\left(a_{1} \cdots\left(e_{k} a_{k}\right) \cdots a_{m} a_{m}^{-1}\right) a_{1} \cdots\left(e_{k} a_{k}\right) \cdots a_{m} \\
\quad\left(\text { by commuting the idempotents } e_{k} \text { and } a_{k-1}^{-1} \cdots a_{1}^{-1} a_{1} \cdots a_{k-1}\right) \\
=\varepsilon\left(a_{1} \cdots\left(e_{k} a_{k}\right) \cdots a_{m} a_{m}^{-1}\right) R(a),
\end{gathered}
$$

by an examination of the various cases. Thus $\varepsilon(A) a=\varepsilon(R(A)) a=\varepsilon(R(A)) R(a)$. 
Now if $A \in \mathbb{Q}$ and $a$ is an associate of $A$ the terminating set $\operatorname{cl}(A)$ of the sequence $L(A), R L(A), \ldots$ has as associate the corresponding word in the sequence $L(a), R L(a), \ldots$ thus obtained. The preceding results are now summarized in

THEOREM 3.3. Let $S$ and $T$ be inverse semigroups and let $\mathscr{Q}$ and $\mathcal{Y}$ be the families of precanonical and canonical sets, respectively, in $S \operatorname{sgp} T$. Then from each member $A$ of Q may be obtained a member $\operatorname{cl}(A)$ of $\mathcal{Y}$ such that $\varepsilon(A)=\varepsilon(\mathrm{cl}(A))$; for each associate a of $A$ there is an associate $x$ (necessarily reduced) of $\operatorname{cl}(A)$ such that $\varepsilon(A) a=\varepsilon(\operatorname{cl}(A)) x$. Hence every element of $S$ inv $T$ is expressible in the form $\varepsilon(X) x$ for some $X \in \mathcal{Y}$ and associate $x$ of $X$. Each idempotent is expressible in the form $\varepsilon(X)$ for some $X \in \mathcal{Y}$.

Since the reduction process relies only on the fact that $S$ and $T$ generate $S$ inv $T$, the following corollary is evident.

COROllary 3.4. Let $W$ be any inverse semigroup generated by $S$ and $T$. Then each element of $W$ is expressible in the form $\varepsilon(X) x$ for some canonical set $X$ and associate $x$ of $X$.

In $\$ 5$ it will be shown that in $S$ inv $T$ the expression $\varepsilon(X) x$ is unique, and that this in fact characterizes the free product amongst inverse semigroups generated by $S$ and $T$ (or isomorphic copies thereof). This will be done by defining a product on the set $F=F(S, T)$ of all ordered pairs $(X, x)$, where $X \in \mathcal{Y}$ and $x$ is an associate of $X$, in such a way that $F$ becomes an inverse semigroup isomorphic with $S$ inv $T$. The first stage, however, is to define a partial order on $\mathcal{O}$ so that it becomes a semilattice isomorphic with $E(S$ inv $T)$, demonstrating the uniqueness of the expression $\varepsilon(X)$ for idempotents of $S \operatorname{inv} T$.

4. An idempotent separating representation of $S$ inv $T$. In this section a representation $\rho$ of $S \operatorname{inv} T$ in $\mathscr{G}_{\mathscr{O}}$, the symmetric inverse semigroup on the set $\mathcal{Y}$, is defined. This representation is then used to define a partial order on $\mathcal{Y}$ in such a way that the image of $S$ inv $T$ in $9_{\text {Oु }}$ consists of partial order isomorphisms, and then to show that, under this partial order, $\mathscr{G}$ is in fact a semilattice isomorphic with $E(S \operatorname{inv} T)$. In fact $\rho$ turns out to be a representation of $S \operatorname{inv} T$ in the Munn semigroup $T_{\mathscr{O}}$ of isomorphisms between principal ideals of $\mathcal{Y}$.

First, if $A \in \mathbb{Q}$ and $s \in S$ let

$$
s \cdot A=\{s \cdot a: a \in A\},
$$

where $s \cdot a$ is the right idempotent word obtained from $a$ by premultiplying by $s$ : thus $s \cdot a=s a(\operatorname{in} S \operatorname{sgp} T)$ except if $a \in E(S)$, when $s \cdot a=s a s^{-1}$.

Now if $A \in \mathcal{Y}, s \in S$ and $s s^{-1} \in A$ define $s^{-1} * A$ by

$$
s^{-1} * A=\left\{\begin{array}{l}
A \quad \text { if } s \text { is idempotent, } \\
\left\{\hat{a}_{1}: a \in A, a_{1}=s\right\} \cup s^{-1} \cdot\left\{a \in A: a_{1} \neq s\right\} \quad \text { otherwise. }
\end{array}\right.
$$

For example if $a=a_{1} \cdots a_{m}$ is a left reduced word, with $m>1$ and $a_{1} \in S$,

$$
a_{1}^{-1} * \operatorname{pre}\left(a_{1} \cdots a_{m}\right)=\left\{a_{1}^{-1} a_{1}\right\} \cup \operatorname{pre}\left(\hat{a}_{1}\right) \text {. }
$$

It may be easily verified that $s^{-1} * A=L\left(s^{-1} \cdot A\right)=\operatorname{cl}\left(s^{-1} \cdot A\right) \in \mathcal{Y}$. 
So far the action $s^{-1} * A$ has only been defined when $s s^{-1} \in A$. We extend the definition as follows. For each $e \in E(S)$ let $\mathscr{Y}_{e}=\left\{A \in \mathcal{Y}: e \geqslant g\right.$ for some $\left.g \in A_{S}\right\}$. Note that the idempotent $g$ is unique, since $A$ has unique last letters. Now for any $s \in S$ and $A \in \mathcal{Y}_{s s^{-1}}$ let $s^{-1} * A=(g s)^{-1} * A$, where $s s^{-1} \geqslant g \in A_{S}$.

Proposition 4.1. For each $s$ in $S$ define $\rho_{s}$ by $A \rho_{s}=s^{-1} * A$ for all $A \in \mathscr{O}_{s s^{-1}}$. Then the map $s \rightarrow \rho_{s}$ is a representation of $S$ in $\mathscr{Q}_{\mathcal{Q}}$.

Proof. To show $\rho_{s} \in \mathscr{Q}_{\mathscr{O}}$ it is sufficient to show that for each $A$ in $\Delta \rho_{s}=\mathscr{O}_{s s^{-1}}$, $A \rho_{s} \rho_{s^{-1}}=A$, for then $\rho_{s^{-1}}=\rho_{s}^{-1}$. If $g$ is the idempotent in $A_{S}$ such that $g \leqslant s s^{-1}$ then by definition $A \rho_{s}=A \rho_{g s}$; note also that $s^{-1} g s=(g s)^{-1} g(g s) \in(g s)^{-1} * A$, and $s^{-1} g s$ $\leqslant s^{-1} s$. Thus $A \rho_{s} \in \Delta \rho_{s^{-1}}$ and $\left(A \rho_{s}\right) \rho_{s^{-1}}=\left(A \rho_{g s}\right) \rho_{(g s)^{-1}}$. Without loss of generality, then, it may be assumed that $s s^{-1} \in A$ (and $s^{-1} s \in A \rho_{s}$ ).

If $s \in E(S)$ then $\left(A \rho_{s}\right) \rho_{s^{-1}}=A \rho_{s^{-1}}=A$. Otherwise

$$
s^{-1} * A=\left\{\hat{a}_{1}: a \in A, a_{1}=s\right\} \cup s^{-1} \cdot\left\{a \in A: a_{1} \neq s\right\}
$$

and

$$
s *\left(s^{-1} * A\right)=s \cdot\left\{\hat{a}_{1}: a \in A, a_{1}=s\right\} \cup\left\{a \in A: a_{1} \neq s\right\}=A .
$$

Before showing that $\rho$ is a morphism, note that if $e, f \in E(S)$ then $\mathscr{Q}_{e} \cap \hat{g}_{f}=\hat{g}_{e f}$ (since $A \in \mathscr{P}_{e} \cap \mathscr{P}_{f}$ if and only if $e \geqslant g$ and $f \geqslant h$ for some $g, h \in A_{S}$ : then $g=h$ since $A$ has unique last letters).

Note also that from the argument above it follows that if $f \leqslant s s^{-1}$ then ${ }_{\mathcal{F}_{f}} \rho_{s}=$ $\mathscr{P}_{s^{-1} f s}$.

Now if $s, u \in S$,

$$
\Delta \rho_{s} \rho_{u}=\left(\mathscr{O}_{s^{-1} s} \cap \mathscr{\mathcal { Y }}_{u u^{-1}}\right) \rho_{s}^{-1}=\mathscr{\mathcal { O }}_{s^{-1} s_{u} u^{-1}} \rho_{s^{-1}}=\mathscr{O}_{(s u)(s u)^{-1}}=\Delta \rho_{s u} .
$$

Let $A \in \Delta \rho_{s u}$, so $(s u)(s u)^{-1} \geqslant g$ for some $g \in A_{s}$. Then $A \rho_{s}=A \rho_{g s}$ and $A \rho_{s u}=A \rho_{g s u}$; also

$$
u u^{-1} \geqslant\left(s^{-1} s\right)\left(u u^{-1}\right) \geqslant s^{-1} g s \in\left(A \rho_{s}\right)_{S},
$$

so $\left(A \rho_{s}\right) \rho_{u}=\left(A \rho_{s}\right) \rho_{s^{-1} g s u}$. But $g s u=(g s)\left(s^{-1} g s u\right)$, so without loss of generality it may be assumed that $s s^{-1}=(g s)(g s)^{-1}=g$ and $u u^{-1}=s^{-1} g s=s^{-1} s$.

If $s \in E(S)$ then $A \rho_{s}=A$ and $\left(A \rho_{s}\right) \rho_{s}=A \rho_{u}=A \rho_{s u}$ (since $\left.u=s^{-1} s u=s u\right)$; the case $u \in E(S)$ is treated similarly. If $s u \in E(S)$ then $u=s^{-1}$ and this case has already been covered. Otherwise

$$
\begin{aligned}
\left(A \rho_{s}\right) \rho_{u}= & u^{-1} \cdot\left\{\hat{a}_{1}: a \in S, a_{1}=s\right\} \cup u^{-1} \cdot\left(s^{-1} \cdot\left\{a \in A: a_{1} \neq s, s^{-1} a_{1} \neq u\right\}\right) \\
& \cup\left\{\hat{a}_{1}: a \in A, a_{1} \neq s, s^{-1} a_{1}=u\right\} .
\end{aligned}
$$

Now if $a_{1}=s, u^{-1}=u^{-1} s^{-1} s=(s u)^{-1} a_{1}$, so

$$
u^{-1} \cdot\left\{\hat{a}_{1}: a \in S, a_{1}=s\right\}=(s u)^{-1} \cdot\left\{a \in A: a_{1}=s\right\} ;
$$

also $s^{-1} a_{1}=u$ if and only if $a_{1}=s u\left(\right.$ since $\left.s \Re a_{1} \Re u\right)$ so

$$
u^{-1} \cdot\left(s^{-1} \cdot\left\{a \in A: a_{1} \neq s, s^{-1} a \neq u\right\}\right)=(s u)^{-1} \cdot\left\{a \in A: a_{1} \neq s u, a_{1} \neq s\right\}
$$


and

$$
\left\{\hat{a}_{1}: a \in A, s_{1} \neq s, s^{-1} a_{1}=u\right\}=\left\{\hat{a}_{1}: a \in A, a_{1}=s u\right\} .
$$

(Note that if $a_{1}=s$ then $a_{1} \neq s u$, and if $a_{1}=s u, a_{1} \neq s$.) Hence $\left(A \rho_{s}\right) \rho_{u}=A \rho_{s u}$.

Therefore the map $s \rightarrow \rho_{s}$ is a morphism of $S$ into $\mathscr{Q}_{\mathscr{Q}}$.

A similar representation $t \rightarrow \rho_{t}$ of $T$ in $\oint_{\mathscr{O}}$ may be defined. From the universal properties of $S \operatorname{inv} T$ it follows that these two representations have a common extension to a representation $\rho$ of $S \operatorname{inv} T$ in $\mathscr{G}_{\mathscr{Q}}$.

EXAMPLE 4.2. If $a=a_{1} \cdots a_{m}$ is a left reduced word, then $a$ may also be considered as an element of $S \operatorname{inv} T$. As noted above,

$$
\operatorname{pre}(a) \rho_{a_{1}}=\left\{a_{1}^{-1} a_{1}\right\} \cup \operatorname{pre}\left(a_{2} \cdots a_{m}\right) \text {. }
$$

Similarly

$$
\operatorname{pre}(a) \rho_{a_{1} a_{2}}=\operatorname{pre}\left(a_{2}^{-1} a_{1}^{-1} a_{1}\right) \cup \operatorname{pre}\left(a_{3} \cdots a_{m}\right)
$$

A simple induction yields

$$
\operatorname{pre}(a) \rho_{a_{1} \cdots a_{1-1}}=\operatorname{pre}\left(a_{i-1}^{-1} \cdots a_{1}^{-1} a_{1}\right) \cup \operatorname{pre}\left(a_{i} \cdots a_{m}\right), \quad 1<i \leqslant m .
$$

Note that $a_{i} a_{i}^{-1} \in \operatorname{pre}(a) \rho_{a_{1} \cdots a_{i-1}}, 1<i \leqslant m$.

This representation is used to define a relation on $\mathcal{O}$ as follows. First, if $a$ is a left reduced word and $B \in \mathcal{Y}$, let $\operatorname{pre}(a) \geqslant B$ if $B \in \mathcal{Y}_{a_{1} a_{1}^{-1}}$ and $B \rho_{a_{1} \cdots a_{i-1}} \in \mathcal{Y}_{a_{i} a_{i}^{-1}}$, $1<i \leqslant m$; equivalently, from the definition of $\rho, \operatorname{pre}(a) \geqslant B$ if and only if there exist idempotents $g_{1}, \ldots, g_{m}$ where $g_{i} \leqslant a_{i} a_{i}^{-1}, 1 \leqslant i \leqslant m, g_{1} \in B$ and $g_{i} \in B \rho_{a_{1} \cdots a_{i-1}}$, $1<i \leqslant m$. Note that

$$
B \rho_{a_{1} \ldots a_{i-1}}=B \rho_{\left(g_{1} a_{1}\right) \cdots\left(g_{i-1} a_{i-1}\right)} .
$$

Now for any $A, B \in \mathcal{Y}$ put $A \geqslant B$ if $\operatorname{pre}(a) \geqslant B$ for all $a \in A$. (Of course $B \leqslant A$ will mean the same as $A \geqslant B$.)

Before showing $\leqslant$ is a partial order on $\mathcal{O}$ it will be shown that $\leqslant$ is "compatible" with the representation $\rho$.

Lemma 4.3. Let $e \in E(S)$ and $A, B \in \mathcal{Y}_{e}$, with $A \geqslant B$. Then $A \rho_{s} \geqslant B \rho_{s}$ for all $s$ Re. Thus for any $x$ in $S \operatorname{inv} T$ for which $A, B \in \Delta \rho_{x}, A \geqslant B$ implies $A \rho_{x} \geqslant B \rho_{x}$.

Proof. Let $h \in B_{S}, g \in A_{S}$, with $h \leqslant g \leqslant e$. Clearly if $s \Re e$, then $g s \Re g$ and $h s=h(g s) \Re h$, so without loss of generality $s s^{-1}=g$.

Let $x \in A \rho_{s}$. There are four cases to consider:

(i) If $x=\hat{a}_{1}$ for some $a=s a_{2} \cdots a_{m} \in A$ then, since pre $(a) \geqslant B,\left(B \rho_{s}\right) \rho_{a_{2} \cdots a_{i-1}}$ $=B \rho_{s a_{2} \cdots a_{i-1}} \in \mathcal{Y}_{a_{i} a_{i}^{-1}}, 1<\mathrm{i} \leqslant m$;

(ii) If $x=s^{-1} s$ then $s^{-1} s \geqslant s^{-1} h s \in B \rho_{s}$;

(iii) If $x=\left(s^{-1} a_{1}\right) a_{2} \cdots a_{m}$ for some $a \in A_{S}$, with $a_{1} \neq s$ (and $\left.l(a)>1\right)$, then $a_{1} a_{1}^{-1}=g \geqslant h$, so

$$
\left(s^{-1} a_{1}\right)\left(s^{-1} a_{1}\right)^{-1}=s^{-1} s \geqslant s^{-1} h s \in B \rho_{s}
$$


and

$$
\left(B \rho_{s}\right) \rho_{\left(s^{-1} a_{1}\right) \cdots a_{i-1}}=\left(B \rho_{s s^{-1} a_{1}}\right) \rho_{a_{2} \ldots a_{i-1}}=B \rho_{a_{1} \ldots a_{i-1}} \in \mathscr{P}_{a_{i} a_{i}^{-1}}, \quad 1<i \leqslant m
$$

(iv) If $x=s^{-1} c_{1} \cdots c_{n}$ for some $c \in A_{T}$, then $s^{-1} s \geqslant s^{-1} h s \in B \rho_{s}$ and

$$
\left(B \rho_{s}\right) \rho_{s^{-1} c_{1} \cdots c_{i-1}}=\left(B \rho_{s s^{-1}}\right) \rho_{c_{1} \cdots c_{i-1}}=B \rho_{c_{1} \cdots c_{i-1}} \in \mathcal{Q}_{c_{i} c_{i}^{-1}}, \quad 1 \leqslant i \leqslant n .
$$

In each case, therefore, $\operatorname{pre}(x) \geqslant B$.

This completes the proof of the first statement. The second follows by induction.

Proposition 4.4. The relation $\leqslant$ is a partial order on $\mathcal{Y}$. Hence $\rho$ is a representation of $S$ inv $T$ by partial order isomorphisms of $\mathcal{Y}$.

Proof. Clearly $\leqslant$ is reflexive. To prove transitivity it is sufficient to consider the case where $a$ is a left reduced word, $B, C \in \mathcal{Y}$ and $\operatorname{pre}(a) \geqslant B, B \geqslant C$. Then $h \leqslant g \leqslant a_{1} a_{1}^{-1}$ for some $h \in C, g \in B$. Therefore $C \in \mathcal{Y}_{a_{1} a_{1}^{-1}}$. For $1<i \leqslant m$, $B \rho_{a_{1} \cdots a_{i-1}} \in \mathcal{O}_{a_{i} a_{i}^{-1}}$ and by the lemma

$$
C \rho_{a_{1} \cdots a_{i-1}} \leqslant B \rho_{a_{1} \cdots a_{i-1}} \text {, so } C \rho_{a_{1} \cdots a_{i-1}} \in \mathcal{Y}_{a_{i} a_{i}^{-1}}
$$

Thus $\operatorname{pre}(a) \geqslant C$.

To show antisymmetry, suppose $A \geqslant B$ and $B \geqslant A$, in $\mathcal{Y}$. Let $a \in A_{S}$, say. There exist idempotents $h_{i} \leqslant g_{i} \leqslant a_{i} a_{i}^{-1}, 1 \leqslant i \leqslant m$, where $h_{1} \in A, g_{1} \in B, h_{i} \in A \rho_{a_{1} \cdots a_{i-1}}$ and $g_{i} \in B \rho_{a_{1} \cdots a_{t-1}}, 1<i \leqslant m$. Since $A$ has unique last letters, $h_{1}=g_{1}=a_{1} a_{1}^{-1}$. Similarly $h_{i}=g_{i}=a_{i} a_{i}^{-1}, 1<i \leqslant m$. Now $a_{m}=a_{m} a_{m}^{-1} \in B \rho_{a_{1} \cdots a_{m-1}}$ so from the definition of $a_{m-1}^{-1} * B \rho_{a_{1} \cdots a_{m-2}}, a_{m-1} a_{m} \in B \rho_{a_{1} \cdots a_{m-2}}$. An induction yields $a_{i} \cdots a_{m}$ $\in B \rho_{a_{1} \cdots a_{i-1}}, 1<i \leqslant m$, so $a_{2} \cdots a_{m} \in B \rho_{a}$, and $a \in B$. Hence $A \subseteq B$ and, similarly, $B \subseteq A$.

The final remark is an immediate consequence of the preceding lemma, for any $\rho_{x}$ is an order isomorphism of $\Delta \rho_{x}$ upon $\nabla \rho_{x}$.

Now for each $A \in \mathcal{Y}$, it is clear that $\operatorname{pre}(a) \geqslant A$ for all $a \in A$ (since $a_{i} a_{i}^{-1} \in$ $\left.A \rho_{a_{1} \cdots a_{i-1}}, 1<i \leqslant m\right)$; moreover if $B \in \mathcal{Y}$ and $\operatorname{pre}(a) \geqslant B$ for all $a \in A$ then $A \geqslant B$, by definition. Thus

Lemma 4.5. For each $A \in \mathcal{Y}, A$ is the greatest lower bound of $\{\operatorname{pre}(a): a \in A\}$. This will be written $A=\wedge\{\operatorname{pre}(a): a \in A\}$.

With each $A \in \mathcal{O}$ there is associated the idempotent $\varepsilon(A)$ defined in $\S 2$. The map $A \rightarrow \varepsilon(A)$ is now related with the representation $\rho$, whence with the partial order on Qु.

LeMmA 4.6. Let $s \in S$ and $A \in \mathcal{Y}_{s s^{-1}}$. Then

$$
\varepsilon\left(A \rho_{s}\right)=s^{-1} \varepsilon(A) s .
$$

Thus for any $x \in S \operatorname{inv} T$ and $A \in \Delta \rho_{x}, \varepsilon\left(A \rho_{x}\right)=x^{-1} \varepsilon(A) x$. 
PRoof. In the first case it may be assumed, without loss of generality, that $s s^{-1} \in A$. (For if $g \in A_{S}, g \leqslant s s^{-1}$, then $g \geqslant \varepsilon(A)$.) Then $A \rho_{s}=s^{-1} * A=\operatorname{cl}\left(s^{-1} \cdot A\right)$, as observed earlier, and

$$
\begin{aligned}
\varepsilon\left(A \rho_{s}\right) & =\varepsilon\left(\mathrm{cl}\left(s^{-1} \cdot A\right)\right)=\varepsilon\left(s^{-1} \cdot A\right) \quad \text { (by Theorem 3.3) } \\
& =s^{-1} \varepsilon(A) s .
\end{aligned}
$$

The second statement is then obvious.

Proposition 4.7. The map $A \rightarrow \varepsilon(A)$, of $\mathcal{Y}$ into $E(S \operatorname{inv} T)$, is order preserving.

Proof. It is clearly sufficient to show $\varepsilon(a)(=\varepsilon(\operatorname{pre}(a))) \geqslant \varepsilon(B)$ whenever pre $(a)$ $\geqslant B$ in $\mathcal{Y}$. In that case $B \rho_{a_{1} \cdots a_{m-1}} \in \mathcal{Y}_{a_{m} a_{m}^{-m}}$, so

$$
a_{m}=a_{m} a_{m}^{-1} \geqslant \varepsilon\left(B \rho_{a_{1} \cdots a_{m-1}}\right),
$$

whence

$$
\begin{aligned}
\left(a_{1} \cdots a_{m-1}\right) a_{m}\left(a_{m-1}^{-1} \cdots a_{1}^{-1}\right) & \geqslant a_{1} \cdots a_{m-1} \varepsilon\left(B \rho_{a_{1} \cdots a_{m-1}}\right) a_{m-1}^{-1} \cdots a_{1}^{-1} \\
& =\varepsilon\left(B \rho_{a_{1} \cdots a_{m-1}} \rho_{a_{m-1}^{-1} \cdots a_{1}^{-1}}\right) \\
& =\varepsilon(B), \text { since } \rho_{a_{i}^{-1}}=\rho_{a_{i}}^{-1}, 1 \leqslant i \leqslant m-1 .
\end{aligned}
$$

Finally the image of $E(\operatorname{Sinv} T)$ in $\Phi_{0 y}$ is established, making use of the following notation: for each $A \in \mathcal{Y}$, denote by $\mathcal{Y}_{A}$ the principal ideal of $(\mathcal{Y}, \leqslant)$ which it generates. (Thus if $e \in E(S) \cup E(T), \mathscr{Y}_{\{e\}}=\mathscr{Y}_{e}$, as already defined.)

Proposition 4.8. Let $A \in \mathcal{Y}$. Then $\Delta \rho_{\varepsilon(A)}=\mathcal{Y}_{A}$, so that $\rho_{\varepsilon(A)}=\iota_{A}$, then identity order automorphism of $\mathcal{Y}_{A}$.

PROOF. First it will be shown that $\Delta \rho_{\varepsilon(a)}=\mathcal{Y}_{\text {pre }(a)}$ for every left reduced word $a$. If $l(a)=1$ then $a \in S \cup T$ and this is just the original definition. Assume the statement is true for all words of length less than $m$, and suppose $l(a)=m>1$. By hypothesis, $\Delta \rho_{\varepsilon\left(\hat{a}_{1}\right)}=\mathcal{O}_{\operatorname{pre}\left(\hat{a}_{1}\right)}$.

Then

$$
\Delta \rho_{\varepsilon(a)}=\left(\Delta \rho_{a_{1}} \cap \nabla \rho_{\varepsilon\left(\hat{a}_{1}\right)}\right) \rho_{a_{1}}^{-1}=\left(\mathcal{Y}_{a_{1} a_{1}^{-1}} \cap \mathcal{Y}_{\operatorname{pr}\left(\hat{a}_{1}\right)}\right) \rho_{a_{1}}^{-1} .
$$

From Example 4.2,

$$
\left\{a_{1} a_{1}^{-1}\right\} \cup \operatorname{pre}\left(\hat{a}_{1}\right)=\operatorname{pre}(a) \rho_{a_{1}},
$$

so by Lemma 4.5 ,

$$
\operatorname{pre}(a) \rho_{a_{1}}=\left\{a_{1} a_{1}^{-1}\right\} \wedge \operatorname{pre}\left(\hat{a}_{1}\right), \text { in } \mathcal{Y}
$$

Thus

$$
\mathscr{Y}_{a_{1} a_{1}^{-1}} \cap \mathcal{Y}_{\operatorname{pre}\left(\hat{a}_{1}\right)}=\mathcal{Y}_{\operatorname{pre}(a) \rho_{a_{1}}}=\left(\mathcal{Y}_{\operatorname{pre}(a)}\right) \rho_{a_{1}},
$$

so

$$
\Delta \rho_{\varepsilon(a)}=\mathcal{O}_{\operatorname{pre}(a)} .
$$


Now for any $A \in \mathcal{Y}, \varepsilon(A)=\prod\{\varepsilon(a): a \in A\}$, so

$$
\rho_{\varepsilon(A)}=\prod\left\{\rho_{\varepsilon(a)}: a \in A\right\}
$$

and

$$
\begin{aligned}
\Delta \rho_{\varepsilon(A)} & =\cap\left\{\Delta \rho_{\varepsilon(a)}: a \in A\right\}=\bigcap\left\{\mathscr{\mathcal { Y }}_{\operatorname{pre}(a)}: a \in A\right\} \\
& =\mathscr{G}_{A}, \quad \text { since } A=\wedge\{\operatorname{pre}(a): a \in A\}, \text { by Lemma } 4.5 .
\end{aligned}
$$

The main result of this section can now be proved.

THEOREM 4.9. Let $S$ and $T$ be inverse semigroups and let $\mathcal{Y}$ be the family of canonical sets in $S \operatorname{sgp} T$, partially ordered by the relation $\leqslant$ defined above. Under the operation $A \wedge B=\operatorname{cl}(A \cup B),(\mathcal{Y}, \leqslant)$ is a semilattice isomorphic with $E(S \operatorname{inv} T)$. The representation $\rho$ is an idempotent separating morphism of $S \operatorname{inv} T$ onto a full inverse subsemigroup of the Munn semigroup $T_{\Theta_{3}}$. Each idempotent $S$ inv $T$ is uniquely expressible in the form $\varepsilon(A)$ for some $A \in \mathcal{Y}$.

Proof. Since each idempotent of $S$ inv $T$ is expressible in the form $\varepsilon(A), A \in \mathcal{O}$, the composition of $\rho \mid E(S$ inv $T)$ with the obvious order isomorphism $\iota_{A} \rightarrow \mathscr{G}_{A} \rightarrow A$ is an order preserving map of $E(S \operatorname{inv} T)$ upon $\mathcal{Y}$. But clearly this composition has as inverse the map $A \rightarrow \varepsilon(A)$, which is also order preserving, by Proposition 4.7. Thus each of these maps is an order isomorphism.

Hence $(\mathcal{Y}, \leqslant)$ is a semilattice, $\rho$ is idempotent separating and represents $S$ by isomorphisms between principal ideals of of (that is, $\rho: S \operatorname{inv} T \rightarrow T_{B}$, the image being clearly full), and the expression $\varepsilon(A)$ is unique.

Finally, for any $A, B$ in $\mathcal{Y}$,

$$
\varepsilon(A) \varepsilon(B)=\varepsilon(A \cup B)=\varepsilon(\operatorname{cl}(A \cup B)),
$$

so $\operatorname{cl}(A \cup B)$ is the greatest lower bound of $A$ and $B$ in ( $\mathcal{Y}, \leqslant)$.

As an immediate consequence it may be observed that any sequence of $L$ and $R$ steps, when applied to a member $A$ of $\mathcal{Q}$, will yield $\operatorname{cl}(A)$. (For the sequence must terminate, and terminate in a member $B$, say, of $\mathscr{Y}$, with $\varepsilon(B)=\varepsilon(A)$, for the same reasons as for the sequence in $\S 2$.)

To complete this section (and to lead to the next) a convenient alternative form for the relation $\leqslant$ on $\mathcal{Y}$ will be given using the following concept. A right idempotent word $b=b_{1} \cdots b_{n}$ is called a pseudomember of $A \in \mathcal{O}$ if $b_{1} b_{1}^{-1} \in A$ and $b_{i} b_{i}^{-1} \in A \rho_{b_{1} \cdots b_{i-1}}, 1<i \leqslant m$. By Example 4.2, any member of $A$ is a pseudomember. In fact a pseudomember of $A$ belongs to $A$ if and only if it is left reduced. (For, if $b$ is left reduced, $b_{n} \in A \rho_{b_{1} \cdots b_{n-1}}$ and by the definition of the action, $b_{n-1} b_{n} \in$ $A \rho_{b_{1} \cdots b_{n-2}}$; a simple induction yields $b \in A$.)

Proposition 4.10. Let $A \in \mathcal{O}$ and let a be a left reduced word. Then $\operatorname{pre}(a) \geqslant A$ if and only if $a$ is above a pseudomember of $A$.

Proof. Suppose pre $(a) \geqslant A$. Then there exist idempotents $g_{1} \in A$ and

$$
g_{i} \in A \rho_{a_{1} \cdots a_{i-1}}=A \rho_{\left(g_{1} a_{1}\right) \cdots\left(g_{i-1} a_{i-1}\right)}, \quad 1<i \leqslant m,
$$


such that $g_{i} \leqslant a_{i} a_{i}^{-1}, 1 \leqslant i \leqslant m$. It is clear that $\left(g_{1} a_{1}\right) \cdots\left(g_{m} a_{m}\right)$ is then a pseudomember of $A$ below $a$.

Conversely, suppose $b=b_{1} \cdots b_{m}$ is a pseudomember of $A$ below $a$. Then $a_{1} a_{1}^{-1} \geqslant b_{1} b_{1}^{-1} \in A$ and for $1<i \leqslant m$,

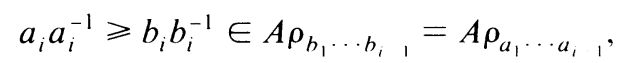

since $b_{j}=b_{j} b_{j}^{-1} a_{j}, 1 \leqslant j \leqslant m$.

On the other hand, associates of $A(\in \mathscr{O})$ ) may be characterized in terms of $\rho$ : $a=a_{1} \cdots a_{m}$ is an associate of $A$ if and only if $a$ is reduced, $a_{1} a_{1}^{-1} \in A$ and $a_{i} a_{i}^{-1} \in A \rho_{a_{1} \cdots a_{1-1}}, 1<i \leqslant m$. (Necessity follows as in Example 4.2; on the other hand if $a_{m} a_{m}^{-1} \in A \rho_{a_{1} \cdots a_{m-1}}$, then since $a_{m}$ and $a_{m-1}$ belong to different factors, $a_{m-1} a_{m} a_{m}^{-1} \in A \rho_{a_{1}} \cdots a_{m-2}$. A straightforward induction yields $a_{1} \cdots a_{m} a_{m}^{-1} \in A$.) Note that if $a$ is an associate of $A$ then $a^{-1}$ is an associate of $A \rho_{a}$ (for $a_{m}^{-1} \cdots a_{1}^{-1} a_{1} \in$ $\left.A \rho_{a}\right)$.

In a similar vein, if $A \in \mathcal{Y}$, a pseudoassociate of $A$ is any word $a=1$ or $a=a_{1} \cdots a_{m}$ with $a_{1} a_{1}^{-1} \in A$ and $a_{i} a_{i}^{-1} \in A \rho_{a_{1} \cdots a_{i-1}}, 1<i \leqslant m$. Thus a pseudoassociate of $A$ is an associate if and only if it is reduced.

5. A faithful representation of $S$ inv $T$. As in $\S 3$, let $F$ (or $F(S, T)$ if there is any possibility of confusion $)=\{(A, a): A \in \mathcal{Y}$ and $a$ is an associate of $A\}$. In this section a product is defined on $F$. A faithful representation $\tau$ of $S \operatorname{inv} T$ in $\mathscr{q}_{F}$ is found and used to show that, under the given product, $F$ is an inverse semigroup isomorphic with $S \operatorname{inv} T$, so that the expression $\varepsilon(A) a$ given in $\S 3$ for elements of $S$ inv $T$ is unique.

In order to define the product and the representation some preliminaries are needed. First, the representation $\rho$ may be extended to $(S \operatorname{inv} T)^{1}$ by letting $\rho_{1}$ be the identity automorphism on $\mathcal{O}$.

Now let $a \in S \operatorname{sgp} T, B \in \mathcal{Y}$ and suppose $a$ is above a pseudoassociate of $B$, that is (cf. Proposition 4.10), there exist idempotents $g_{1} \in B$ and $g_{i} \in B \rho_{a_{1} \ldots a_{t-1}}, 1<i \leqslant$ $m$, such that $g_{i} \leqslant a_{i} a_{i}^{-1}, 1 \leqslant i \leqslant m$. Define $\overline{a_{B}}$ (or just $\bar{a}$ when the context is clear) $b y$

$$
\bar{a}_{B}=\overline{\left(g_{1} a_{1}\right) \cdots\left(g_{m} a_{m}\right)} \text {, }
$$

the reduced word obtained from (the pseudoassociate) $\left(g_{1} a_{1}\right) \cdots\left(g_{m} a_{m}\right)$ by deleting idempotents. Formally, $\bar{a}_{B}$ is the last member of the sequence $b^{(0)}, b^{(1)}, \ldots$, where $b^{(0)}=\left(g_{1} a_{1}\right) \cdots\left(g_{m} a_{m}\right)$, and if $b^{(i-1)}$ is not reduced, $b^{(i)}$ is the word obtained from $b^{(i-1)}$ by deleting the first idempotent letter (so $b^{(i)}=1$ if $b^{(i-1)}$ consists of a single idempotent). If $a=1$ put $\bar{a}_{B}=1$.

A situation frequently encountered in the sequel will be that of two nonempty reduced words $a=a_{1} \cdots a_{m}$ and $b=b_{1} \cdots b_{n}$, say, and a member $C$ of $\mathcal{Y}$ such that $\operatorname{pre}\left(a a_{m}^{-1}\right) \geqslant C$ and $\operatorname{pre}\left(b b_{n}^{-1}\right) \geqslant C \rho_{a}$. Then it is easily verified that $a b(\operatorname{in} S \operatorname{sgp} T)$ is above a pseudoassociate of $C$.

Define a product on $F$ by the rule

$$
(A, a)(B, b)=(C, c), \text { where } C=\left(A \rho_{a} \wedge B\right) \rho_{a}^{-1} \text { and } c=(\overline{a b})_{C} .
$$


To show this is well defined observe first that (if $a \neq 1) A \rho_{a} \wedge B \leqslant A \rho_{a} \leqslant$ pre $\left(a^{-1} a_{1}\right)$, so

$$
A \rho_{a} \wedge B \in \mathcal{Y}_{\operatorname{pre}\left(a^{-1} a_{1}\right)}=\Delta \rho_{\varepsilon\left(a^{-1} a_{1}\right)}=\Delta \rho_{a^{-1} a_{1}}=\Delta \rho_{a^{-1}}=\Delta \rho_{a}^{-1}
$$

(since $a^{-1} a_{1}$ (R $a^{-1}$ in $S$ inv $T$ ), and thus $C$ is well defined. Further, $C \rho_{a}=A \rho_{a} \wedge B$, so $C \rho_{a} \leqslant B \leqslant \operatorname{pre}\left(b b_{n}^{-1}\right)$ and $C \leqslant A \leqslant \operatorname{pre}\left(a a_{m}^{-1}\right)$, so the situation described above arises and $c$ is defined. (The cases when $a=1$ or $b=1$ are simpler.) To show $(\overline{a b})_{C}$ is indeed an associate of $C$ the following lemma suffices.

LEMmA 5.1. Let $A \in \mathcal{Y}$ and suppose $a=a_{1} \cdots a_{m}$ is a pseudoassociate of $A$. Then $\bar{a}$ is an associate of $A$ and $\varepsilon(A) a=\varepsilon(A) \bar{a}$. Thus if $a$ is above a pseudoassociate of $A, \bar{a}_{A}$ is an associate of $A$ and $\varepsilon(A)=\varepsilon(A) \bar{a}_{A}$. Also $A \rho_{a}=A \rho_{\bar{a}_{A}}$.

Proof. If $a$ is already reduced then $\bar{a}=a$ and, from the closing remarks of the previous section, $a$ is an associate of $A$. Suppose $a$ is not reduced, and $a_{k}$ is the first idempotent letter of $a$. If $m=1$ then $\bar{a}=1$, an associate of $A, \varepsilon(A) a=\varepsilon(A)$ (since $a=a_{1} a_{1}^{-1} \in A$ ) and $A \rho_{a}=A$. Otherwise let $b$ be the word obtained from $a$ by deleting $a_{k}$. It is sufficient (proceeding inductively) to show that $b$ is again a pseudoassociate of $A$, that $\varepsilon(A) a=\varepsilon(A) b$ and $A \rho_{a}=A \rho_{b}$.

Suppose $1<k<m$ : then $b=a_{1} \cdots\left(a_{k-1} a_{k+1}\right) \cdots a_{m}=b_{1} \cdots b_{m-2}$, where $b_{j}$ $=a_{j}$ for $j<k-1, b_{k-1}=a_{k-1} a_{k+1}$ and $b_{j}=a_{j+2}$ for $j>k-1$ (if $m>k+1$ ). For $j<k-1, b_{j} b_{j}^{-1}$ obviously has the requisite property. Next, consider $b_{k-1} b_{k-1}^{-1}$ $=a_{k-1} a_{k+1} a_{k+1}^{-1} a_{k-1}^{-1}$. Now, by assumption,

$$
a_{k+1} a_{k+1}^{-1} \in A \rho_{a_{1} \cdots a_{k-1}} \rho_{a_{k}}=A \rho_{a_{1} \cdots a_{k-1}} .
$$

But this set also contains $a_{k-1}^{-1} a_{k-1}$, so

$$
a_{k+1} a_{k+1}^{-1}=a_{k-1}^{-1} a_{k-1} \quad \text { and } \quad b_{k-1} b_{k-1}^{-1}=a_{k-1} a_{k-1}^{-1} \in A \rho_{a_{1} \cdots a_{k-2}}=A \rho_{b_{1} \cdots b_{k-2}} .
$$

For $j>k-1$,

$$
\begin{aligned}
b_{j} b_{j}^{-1} & =a_{j+2} a_{j+2}^{-1} \in A \rho_{a_{1} \cdots a_{k-1}} \rho_{a_{k}} \rho_{a_{k+1} \cdots a_{j+1}} \\
& =A \rho_{a_{1} \cdots\left(a_{k-1} a_{k+1}\right) \cdots a_{j+1}}=A \rho_{b_{1} \cdots b_{j-1}} .
\end{aligned}
$$

Therefore $b$ is again a pseudoassociate of $A$. Observe also that $a_{1} \cdots a_{k-1} a_{k}$ is a pseudomember of $A$ (since $a_{k}$ is idempotent) and left reduced (since $a_{1}, \ldots, a_{k-1}$ are nonidempotent), whence $a_{1} \cdots a_{k-1} a_{k} \in A$. Therefore

$$
\varepsilon(A)=\varepsilon(A) a_{1} \cdots a_{k-1} a_{k} a_{k-1}^{-1} \cdots a_{1}^{-1}
$$

and

$$
\varepsilon(A) a_{1} \cdots a_{k-1}=\varepsilon(A) a_{1} \cdots a_{k-1} a_{k}, \quad \text { so } \quad \varepsilon(A) b=\varepsilon(A) a .
$$

But then, using Lemma 4.6,

$$
\varepsilon\left(A \rho_{a}\right)=a^{-1} \varepsilon(A) a=b^{-1} \varepsilon(A) b=\varepsilon\left(A \rho_{b}\right),
$$


so $A \rho_{a}=A \rho_{b}$, by the uniqueness of the representation.

The cases $k=1$ and $k=m$ are straightforward.

Proposition 5.2. For each $s \in S$ let $\tau_{s}$ be the partial transformation of $F$ defined by

$$
(A, a) \tau_{s}=\left(A,(\overline{a s})_{A}\right), \text { for all }(A, a) \in F \text { with } A \rho_{a} \in \mathcal{Y}_{s s^{-1}} .
$$

Then $s \rightarrow \tau_{s}$ is a representation of $S$ in the symmetric inverse semigroup $q_{F}$ on $F$.

REMARK. Before proving this proposition a more explicit description of $\tau_{s}$ will be given. If $(A, a) \in \Delta \tau_{s}$ let $g \in A \rho_{a}, g \leqslant s s^{-1}$. It is clear that $\overline{(\overline{a s})_{A}}=\overline{a(g s)}$, where $g s \Re g$. (Thus $(A, a) \tau_{s}=(A, a) \tau_{g s}$.) Since $a$ is reduced it follows that at most the last letter of $a(g s)$ may be idempotent. Thus

$$
(\overline{a s})_{A}= \begin{cases}a_{1} \cdots a_{m-1} & \text { if } a_{m}=(g s)^{-1} \text { and } m>1 \\ 1 & \text { if } a=a_{m}=(g s)^{-1} \\ a(g s) & \text { in }(S \operatorname{sgp} T)^{1}, \text { otherwise }\end{cases}
$$

Throughout the sequel, $(\overline{a s})_{A}$ will be abbreviated to $\overline{a s}$.

Proof of the Proposition. By virtue of the remarks preceding the definition of the product on $F, a(g s)$ is a pseudoassociate of $A$ and so, by the lemma, $(A, a) \tau_{s} \in F$. Also $A \rho_{\overline{a s}}=A \rho_{a s} \in \mathscr{Y}_{s s^{-1}} \rho_{s}=\mathscr{Y}_{s^{-1} s}$, that is, $(A, a) \tau_{s} \in \Delta \tau_{s^{-1}}$. From the description given above it is easily verified that $\overline{(a s) s^{-1}}=a$, so $\tau_{s} \tau_{s^{-1}}$ is the identity on $\Delta \tau_{s}$. Hence $\tau_{s^{-1}}=\tau_{s}^{-1}$ and $\tau_{s} \in \mathscr{G}_{F}$.

Let $s, u \in S$. Then

$$
\begin{aligned}
\Delta \tau_{s} \tau_{u} & =\left\{(A, a) \in F: A \rho_{a} \in \Delta \rho_{s} \text { and } A \rho_{\overline{a s}}\left(=A \rho_{a s}\right) \in \Delta \rho_{u}\right\} \\
& =\left\{(A, a) \in F: A \rho_{a s} \in \nabla \rho_{s} \cap \Delta \rho_{u}\right\} \\
& =\left\{(A, a) \in F: A \rho_{a} \in\left(\nabla \rho_{s} \cap \Delta \rho_{u}\right) \rho_{s}^{-1}=\Delta \rho_{s u}\right\} \\
& =\Delta \tau_{s u} .
\end{aligned}
$$

It is once again routine to verify that in each case $\overline{(\overline{a s}) u}=\overline{a(s u)}$. (Note that, as in the proof of Proposition 4.1, it is sufficient to assume that $s s^{-1}=(s u)(s u)^{-1} \in A \rho_{a}$ and $u u^{-1}=s^{-1} s \in A \rho_{a s}$.) Thus $\tau_{s} \tau_{u}=\tau_{s u}$ and the map $s \rightarrow \tau_{s}$ is a morphism.

A similar representation $t \rightarrow \tau_{t}$ of $T$ in $\oint_{F}$ may be defined and a common extension to a representation $\tau$ of $S$ inv $T$ in $\mathscr{G}_{F}$ therefore exists. The image of $S$ inv $T$ in $\mathscr{G}_{F}$ will now be found.

Proposition 5.3. For any $a \in S \operatorname{inv} T$,

$$
\Delta \tau_{a}=\left\{(B, b) \in F: B \rho_{b} \in \Delta \rho_{a}\right\} .
$$

In particular if $A \in \mathcal{Y}, \Delta \tau_{\varepsilon(A)}=\left\{(B, b) \in F: B \rho_{b} \in \mathcal{Y}_{A}\right\}$. If $a$ is an associate of $A$, then for all such $(B, b)$,

$$
(B, b) \tau_{\varepsilon(A) a}=(B, \overline{b a}) .
$$


Proof. If $l(a)=1$ ( $a$ being considered as an element of $S \operatorname{sgp} T$ ) the first statement is obvious. Assume it is true for all words of length less than $m>1$, and let $a=a_{1} \cdots a_{m}$. Put $y=a_{2} \cdots a_{m}$. Then

$$
\begin{aligned}
\Delta \tau_{a} & =\Delta \tau_{a_{i}} \tau_{y}=\left\{(B, b) \in F: B \rho_{b} \in \Delta \rho_{a_{1}} \text { and } B \rho_{b a_{1}} \in \Delta \rho_{y}\right\} \quad \text { (by hypothesis) } \\
& =\left\{(B, b) \in F: B \rho_{b a_{1}} \in \nabla \rho_{a_{1}} \cap \Delta \rho_{y}\right\} \\
& =\left\{(B, b) \in F: B \rho_{b}\left(=B \rho_{b a_{1}} \rho_{a_{1}}^{-1}\right) \in\left(\nabla \rho_{a_{1}} \cap \Delta \rho_{y}\right) \rho_{a_{1}}^{-1}\right\} \\
& =\left\{(B, b) \in F: B \rho_{b} \in \Delta \rho_{a_{1}} \rho_{y}=\Delta \rho_{a}\right\},
\end{aligned}
$$

and the proof of the first statement is completed by induction. The second statement is immediate from Proposition 4.8.

If $a$ is an associate of $A$ then $\varepsilon(A) a a^{-1}=\varepsilon(A)$, so $\Delta \tau_{\varepsilon(A) a}=\Delta \tau_{\varepsilon(A)}$. For $(B, b) \in F$, with $B \rho_{b} \in \mathscr{\vartheta}_{A}$,

$$
\begin{aligned}
(B, b) \tau_{\varepsilon(A) a} & =(B, b) \tau_{a}, \quad \text { since } \tau_{\varepsilon(A)} \text { is the identity on its domain, } \\
& =(B, b) \tau_{a_{1}} \tau_{a_{2}} \cdots \tau_{a_{m}}=(B, b) \tau_{g_{1} a_{1}} \tau_{g_{2} a_{2}} \cdots \tau_{g_{m} a_{m}},
\end{aligned}
$$

where $g_{1} \in B, g_{i} \in B \rho_{a_{1} \cdots a_{t-1}}, 1<i \leqslant m$. and $g_{i} \leqslant a_{i} a_{i}^{-1}, 1 \leqslant i \leqslant m$. Since $b$ is reduced, and using the fact that in the formation of $\overline{b\left(g_{1} a_{1}\right) \cdots\left(g_{m} a_{m}\right)}$ idempotent letters, if any, are deleted "from the front", it is clear that

$$
(B, b) \tau_{a}=\left(B, \overline{b\left(g_{1} a_{1}\right) \cdots\left(g_{m} a_{m}\right)}\right)=(B, \overline{b a}) .
$$

Denote by $\psi$ the map of $F$ into $S$ inv $T$ which takes $(A, a)$ to $\varepsilon(A) a$. The main result of this section can now be proved.

THEOREM 5.4. Let $S$ and $T$ be inverse semigroups and let $F$ be the set of ordered pairs $(A, a)$, where $A$ is a canonical set in $S \operatorname{sgp} T$ and $a$ is an associate of $A$. Under the product defined above, $F$ is an inverse semigroup isomorphic, under $\psi$, with $S$ inv $T$. Each element of $S$ inv $T$ is uniquely expressible in the form $\varepsilon(A)$ a, for some canonical set $A$ and associate $a$ of $A$.

Proof. By Theorem 3.3, $\psi$ is surjective. To show $\psi$ is a bijection, suppose $(A, a),(B, b) \in F$ and $\varepsilon(A) a=\varepsilon(B) b$. Then

$$
\varepsilon(A)=(\varepsilon(A) a)(\varepsilon(A) a)^{-1}=(\varepsilon(B) b)(\varepsilon(B) b)^{-1}=\varepsilon(B),
$$

so $A=B$ by Theorem 4.9. Thus both $a$ and $b$ are associates of $A$. Now by the previous proposition $\left(A \rho_{a}, a^{-1}\right) \in \Delta \tau_{\varepsilon(A)}$,

$$
\left(A \rho_{a}, a^{-1}\right) \tau_{\varepsilon(A) a}=\left(A \rho_{a}, \overline{a^{-1} a}\right)=\left(A \rho_{a}, 1\right), \quad\left(A \rho_{a}, a^{-1}\right) \tau_{\varepsilon(A) b}=\left(A \rho_{a}, \overline{a^{-1} b}\right) .
$$

Thus the product $a^{-1} b$ in $(S \operatorname{sgp} T)^{1}$ reduces to the empty word. Clearly, then, $a=1$ if and only if $b=1$; on the other hand if $a=a_{1} \cdots a_{m}$ then $b=b_{1} \cdots b_{m}$ and $a_{i}^{-1} b_{i}$ is idempotent for $1 \leqslant i \leqslant m$. But since $a_{1}$ and $b_{1}$ both belong to $S$, or to $T, a_{1} \Re b_{1}$, whence $a_{1}=b_{1}$. By induction $a_{i}=b_{i}, 1 \leqslant i \leqslant m$, that is, $a=b$. 
Hence $\psi$ is a bijection and the expression $\varepsilon(A) a$ is unique. Finally, let $(A, a)$, $(B, b) \in F$. Then

$$
\begin{aligned}
((A, a)(B, b)) \psi & =\left(\left(A \rho_{a} \wedge B\right) \rho_{a}^{-1}, \overline{a b}\right) \psi=\varepsilon\left(\left(A \rho_{a} \wedge B\right) \rho_{a}^{-1}\right) \overline{a b} \\
& =\varepsilon\left(\left(A \rho_{a} \wedge B\right) \rho_{a}^{-1}\right) a b, \quad \text { by Lemma } 5.1 \\
& =a \varepsilon\left(A \rho_{a} \wedge B\right) a^{-1} a b, \quad \text { by Lemma } 4.6 \\
& =a \varepsilon\left(A \rho_{a}\right) \varepsilon(B) a^{-1} a b, \quad \text { by Theorem } 4.9 \\
& =a a^{-1} \varepsilon(A) a \varepsilon(B) a^{-1} a b, \quad \text { by Lemma } 4.6 \text { again } \\
& =(\varepsilon(A) a)(\varepsilon(B) b)=(A, a) \psi(B, b) \psi
\end{aligned}
$$

Therefore $\psi$ is an isomorphism, whence $(R, \cdot)$ is an inverse semigroup isomorphic with $S$ inv $T$.

Under the isomorphism $\psi^{-1}$, each $s$ in $S$ clearly corresponds to $\left(\left\{s s^{-1}\right\}, s\right)$, if $s$ is nonidempotent, or to $\left(\left\{s S^{-1}\right\}, 1\right)$ otherwise (and similarly for $\left.T\right)$. Thus $(S \cup T) \psi^{-1}$ $=\{(A, a) \in F:|A|=1\}$.

At this point it is appropriate to give an alternative form for the first component in the product formula for $F$ in terms of the reduction operator.

Proposition 5.5. If $(A, a),(B, b) \in F$ then

$$
(A, a)(B, b)=(\operatorname{cl}(A \cup a \cdot B), \overline{a b}) .
$$

ProOF. By arguments used previously

$$
\begin{aligned}
\varepsilon\left(\left(A \rho_{a} \wedge B\right) \rho_{a}^{-1}\right) & =\varepsilon(A) a \varepsilon(B) a^{-1}=\varepsilon(A) \varepsilon(a \cdot B) \\
& =\varepsilon(A \cup a \cdot B)=\varepsilon(\operatorname{cl}(A \cup a \cdot B)),
\end{aligned}
$$

the uniqueness of the $\varepsilon$ form yielding the result.

In Corollary 3.4 it was shown that in any inverse semigroup $W$ generated by $S$ and $T$, each element is expressible in the form $\varepsilon(A) a$ for some $(A, a) \in F$. On the other hand if this expression is unique for each element of $W$ then the natural morphism of $S$ inv $T$ upon $W$ (extending the injections $S \rightarrow W, T \rightarrow W$ ) is clearly a bijection, yielding the following

Proposition 5.6. Suppose $W$ is an inverse semigroup generated by $S$ and $T$. If each element of $W$ is uniquely expressible in the form $\varepsilon(A) a$, where $A \in \mathcal{Y}$ and $a$ is an associate of $A$, then $W \cong S \operatorname{inv} T$.

COROllary 5.7. Let $U$ and $V$ be inverse subsemigroups of $S$ and $T$ respectively. Then the inverse subsemigroup $W$ of $S$ inv $T$ generated by $U$ and $V$ is isomorphic with $U$ inv $V$.

Proof. By Corollary 3.4 every element of $W$ is expressible in the form $\varepsilon(A) a$, where $A \in \mathcal{O}(U, V)$ and $a$ is an associate of $A$ (and a reduced word in $\left.(U \operatorname{sgp} V)^{\mathrm{l}}\right)$. But clearly $\mathcal{Y}(U, V) \subseteq \mathcal{Y}(S, T)$ and $a$ is also reduced in $(S \operatorname{sgp} T)^{1}$. Thus $(A, a) \in F$ and the expression is unique. Hence $W \cong U$ inv $V$.

This corollary was first obtained by T. E. Hall [2, Theorem 3.10] as a consequence of the amalgamation property for inverse semigroups. 
6. Structural properties of $S$ inv $T$. The isomorphism of $S$ inv $T$ with $F$ will now be used to examine its structure. First some elementary properties are listed. (In (iv), $\mu$ is the greatest idempotent separating congruence (see [3, Theorem V.3.2]).)

TheOREM 6.1. Let $(A, a),(B, b) \in F$. Then

(i) $(A, a)^{-1}=\left(A \rho_{a}, a^{-1}\right)$;

(ii) $(A, a)$ is idempotent if and only if $a=1 ;(A, 1) \geqslant(B, 1)$ if and only if $A \geqslant B$;

(iii) $(A, a) \mathcal{R}(B, b)$ iff $A=B$;

$(A, a) \mathcal{L}(B, b)$ iff $A \rho_{a}=B \rho_{b}$;

$(A, 1) \mathcal{D}(B, 1)$ iff $A \rho_{a}=B$ for some associate a of $A$, and

$(A, a) \mathcal{H}(A, 1)$ iff $A \rho_{a}=A$;

(iv) $(A, a) \mu(A, 1)$ iff $C \rho_{a}=C$ for all $C \leqslant A$;

(v) $(A, a) \geqslant(B, b)$ iff $A \geqslant B$ and $\overline{a_{B}}=b$.

Proof. (i) That $\left(A \rho_{a}, a^{-1}\right) \in F$ has already been observed. Now

$$
\left(A \rho_{a}, a^{-1}\right) \psi=\varepsilon\left(A \rho_{a}\right) a^{-1}=a^{-1} \varepsilon(A) a a^{-1}=a^{-1} \varepsilon(A)=((A, a) \psi)^{-1},
$$

so $\left(A \rho_{a}, a^{-1}\right)=(A, a)^{-1}$;

(ii) and (iii) are clear;

(iv) By definition $(A, a) \mu(A, 1)$ if and only if $(A, a)^{-1}(C, 1)(A, a)=(C, 1)$ for all $C \leqslant A$. But for $C \leqslant A$,

$$
\begin{aligned}
(A, a)^{-1}(C, 1)(A, a) & =\left(A \rho_{a}, a^{-1}\right)(C, 1)(A, a)=\left((A \wedge C) \rho_{a}, \overline{a^{-1}}\right)(A, a) \\
& =\left(C \rho_{a}, \overline{a^{-1}}\right)(A, a)=\left((C \wedge A) \rho_{a}, 1\right)=\left(C \rho_{a}, 1\right),
\end{aligned}
$$

yielding the required result;

(v) If $(A, a) \geqslant(B, b)$ then $(A, 1) \geqslant(B, 1)$, so $A \geqslant B$. Also $(B, b)=(B, 1)(A, a)$ $=\left(B, \overline{a_{B}}\right)$, so $\overline{a_{B}}=b$. The converse is similar.

Green's relation $\mathcal{G}$ has been omitted because, as will be shown in Theorem 6.4, outside $S$ and $T, S$ inv $T$ is completely semisimple, so $\mathscr{D}=\mathscr{g}$. Two technical lemmas are first required for the proof of that theorem.

Lemma 6.2. Let $A \in \mathcal{O},|A| \neq 1$. There are only finitely many elements $s$ of $S$ for which $s^{-1} \in A$ and $A \rho_{s} \leqslant A$. Thus for no $s$ in $S$ is $A \rho_{s}<A$.

Proof. Suppose $A \rho_{s} \leqslant A$, with $s s^{-1} \in A, s \neq s s^{-1}$. Now since $|A| \neq 1$, either $A_{T}$ is nonempty or $A_{S}$ contains a word of length 2 .

In the former case, suppose $f \in A_{T}$. Then $f \geqslant g$ for some $g \in\left(A \rho_{s}\right)_{T}$, whence (from the definition of $A \rho_{s}$ as $s^{-1} * A$ ), sg $\in A_{S}$. But $A$ is finite, so only finitely many such $s$ are possible.

In the latter case, suppose $a_{1} a_{2} \in A_{S}$. Then since $A \geqslant A \rho_{s}, a_{2} a_{2}^{-1} \geqslant h$ for some $h \in\left(A \rho_{s} \rho_{a_{1}}\right)_{T}=\left(A \rho_{s} \rho_{s^{-1} s a_{1}}\right)_{T}$. If $s^{-1} s a_{1} \in E(S)$ then $h \in\left(A \rho_{s}\right)_{T}$ and the result follows as above; if $s^{-1} s a_{1} \notin E(S)$ then $\left(s^{-1} s a_{1}\right) h \in A \rho_{s}$, whence $\left(s a_{1}\right) h \in A$. But $s s^{-1} \in A_{S}$ and $s^{-1} s \in\left(A \rho_{s}\right)_{s}$ so $s s^{-1} \geqslant s^{-1} s$. Thus $s=s\left(s^{-1} s\right)=s\left(s s^{-1}\right)=\left(s a_{1}\right) a_{1}^{-1}$. But there are only finitely many choices for $s a_{1}$ and for $a_{1}$, and thus for $s$. 
Suppose $A \rho_{s}<A$. Then from (ii) and (iii) of Theorem 6.1, $(A, s)$ generates a bicyclic semigroup. But $A \rho_{s^{n}}<A$ for all $n \geqslant 1$, so from the first part of the proof $s^{n}=s^{k}$ for some $k \neq n$, in which case $(A, s)^{k}=\left(A, s^{k}\right) \doteq\left(A, s^{n}\right)=(A, s)^{n}$, which is impossible in a bicyclic semigroup.

LEMMA 6.3. Let $A \in \mathcal{Y}$ and suppose $a$ is an associate of $A$, of length $m$ greater than one, such that $A \rho_{a} \leqslant A$ (whence $A \rho_{a^{n}} \leqslant A$ for all $\left.n \geqslant 1\right)$. For each $n \geqslant 1$ let $h_{1}^{(n)} \in A \rho_{a^{n}}$, $h_{i}^{(n)} \in A \rho_{a^{n} a_{1} \cdots a_{i-1}}, 1<i \leqslant m$, be idempotents such that, for each $i$,

$$
h_{i}^{(n)} \leqslant h_{i}^{(n-1)} \leqslant \cdots \leqslant h_{i}^{(1)} \leqslant h_{i}^{(0)}=a_{i} a_{i}^{-1}
$$

put $a^{(n)}=\left(h_{1}^{(n)} a_{1}\right) \cdots\left(h_{m}^{(n)} a_{m}\right)$ and suppose each $a^{(n)}$ is reduced.

Then $m$ is odd and for some $n \geqslant 1$ cancellation "reaches the central term", that is ( putting $M=(m+1) / 2), h_{m-i+1}^{(n)} a_{m-i+1} h_{i}^{(n+1)} a_{i}$ is idempotent for $1 \leqslant i \leqslant M-1$ and

$$
\overline{a^{(n)} a^{(n+1)}}=\overline{\left(h_{1}^{(n)} a_{1}\right) \cdots\left(h_{M}^{(n)} a_{M} h_{M}^{(n+1)} a_{M}\right) \cdots\left(h_{m}^{(n+1)} a_{m}\right)},
$$

so has length at most $m$.

Proof. By assumption $(A, a)$ generates either a subgroup or a bicyclic subsemigroup of $F$. In either case $(A, a)^{n}=\left(A, \overline{a^{n}}\right)$, so for each $n, \overline{a^{n}}\left(=\overline{a a^{(1)} \cdots a^{(n-1)}}\right)$ is an associate of $A$. Clearly the lengths of these words must therefore be bounded and so cancellation must occur. In particular $m$ cannot be even and for some $k \geqslant 0$, $h_{m}^{(k)} a_{m} h_{1}^{(k+1)} a_{1}$ is idempotent. Suppose cancellation never reaches the central term: then there is a largest positive integer $l$, say, $l<M-1$, such that for some $r \geqslant 0$, $h_{m-i+1}^{(r)} a_{m-i+1} h_{i}^{(r+1)} a_{i}$ is idempotent for $1 \leqslant i \leqslant l$. Note that this remains true for all $s \geqslant r$ by the choice of the idempotents $h_{i}^{(j)}$.

In fact without loss of generality it may be assumed that $r=0$, for

$$
(A, a)^{-r}(A, a)^{r+1}=\left(A \rho_{a^{r}}, 1\right)(A, a)=\left(A \rho_{a^{r}}, \overline{a^{(r)}}\right)=\left(A \rho_{a^{r}}, a^{(r)}\right),
$$

also generates a bicyclic semigroup and for each $n$,

$$
\left(A \rho_{a^{r}}, a^{(r)}\right)^{n}=\left(A \rho_{a^{r}}, \overline{a^{(r)} a^{(r+1)} \cdots a^{(n-1)}}\right) .
$$

Then $\overline{a a^{(1)}}=a_{1} \cdots\left(a_{m-l} h_{l+1}^{(1)} a_{l+1}\right) \cdots\left(h_{m}^{(1)} a_{m}\right)$, of length $(m-l)+(m-l-1)$ $=2 m-2 l-1>m$. Similarly

$$
\overline{a a^{(1)} a^{(2)}}=a_{1} \cdots\left(a_{m-l} h_{l+1}^{(1)} a_{l+1}\right) \cdots\left(h_{m-l}^{(1)} a_{m-l} h_{l+1}^{(2)} a_{l+1}^{(2)}\right) \cdots\left(h_{m}^{(2)} a_{m}\right)
$$

(since $l+1<M<m-l)$, of length $(m-l)+(m-2 l-1)+(m-l-1)>$ $2 m-2 l-1$. By induction,

$$
\begin{aligned}
l \overline{\left(a^{n}\right)} & =l \overline{\left(a a^{(1)} \cdots a^{(n-1)}\right)}=l \overline{\left(a a^{(1)} \cdots a^{(n-2)}\right)}+(m-2 l-1) \\
& >l \overline{\left(a a^{(1)} \cdots a^{(n-2)}\right)}=l \overline{\left(a^{n-1}\right)}, \quad \text { for all } n \geqslant 2 .
\end{aligned}
$$

This contradicts the bound on the lengths of the words $\overline{a^{n}}$.

Hence for some $n \geqslant 1, h_{m-i+1}^{(n)} a_{m-i+1} h_{i}^{(n+1)} a_{i}$ is idempotent for $1 \leqslant i \leqslant M-1$. 
TheOrem 6.4. Any bicyclic subsemigroup of $S \operatorname{inv} T$ belongs to $S$ or to $T$. Hence the ideal ( $S$ inv $T) \backslash(S \cup T)$ is completely semisimple.

Proof. Suppose $(A, a)$ generates a bicyclic subsemigroup of $F$, where $|A| \neq 1$.

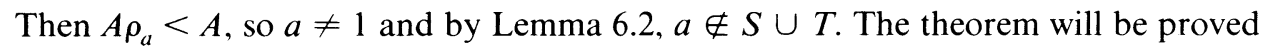
by induction on $l(a)$. Suppose for no $(A, a)$ in $F$, with $l(a)<m(>1)$, is $A \rho_{a}<A$. Let $a=a_{1} \cdots a_{m}$ and use the notation of Lemma 6.3.

For any $n>1,\left(A \rho_{a^{n}}, \overline{a^{(n)}}\right)$ also generates a bicyclic subsemigroup of $F$ (since $\left.\left(A \rho_{a^{n}}, \overline{a^{(n)}}\right)=(A, a)^{-n}(A, a)^{n+1}\right)$. If $a^{(n)}$ is not reduced then $l\left(a^{(n)}\right)<m$, contradicting the induction hypothesis. Thus the hypotheses of Lemma 6.3 are satisfied and, as in the proof of that lemma, it may be assumed that for all $s \geqslant 0$,

$$
h_{m-i+1}^{(s)} a_{m-i+1} h_{i}^{(s+1)} a_{i} \text { is idempotent for } 1 \leqslant i \leqslant M-1,
$$

and

$$
\overline{a^{(s)} a^{(s+1)}}=\overline{\left(h_{1}^{(s)} a_{1}\right) \cdots\left(h_{M}^{(s)} a_{M} h_{M}^{(s+1)} a_{M}\right) \cdots\left(h_{m}^{(s+1)} a_{m}\right)} .
$$

But cancellation reaches no further than the central term, for if $h_{M}^{(s)} a_{M} h_{M}^{(s+1)} a_{M}$ is idempotent, $l \overline{\left(a^{(s)} a^{(s+1)}\right)}<m$, which again yields a contradiction, for

$$
(A, a)^{-s}(A, a)^{s+2}=\left(A \rho_{a^{s}}, \overline{a^{(s)} a^{(s+1)}}\right)
$$

again generates a bicyclic subsemigroup. Hence for each $n \geqslant 1$,

$$
\begin{aligned}
\overline{a^{n}} & =\overline{a a^{(1)} \cdots a^{(n-1)}} \\
& =a_{1} \cdots a_{M-1}\left(a_{M} h_{M}^{(1)} a_{M} h_{M}^{(2)} a_{M} \cdots h_{M}^{(n-1)} a_{M}\right)\left(h_{M+1}^{(n-1)} a_{M+1}\right) \cdots\left(h_{m}^{(n-1)} a_{m}\right) .
\end{aligned}
$$

Now $h_{M}^{(1)}$ and $a_{M-1}^{-1} a_{M}$ both belong to $A \rho_{a_{1} \cdots a_{M-1}}$, so

$$
h_{M}^{(1)}=a_{M}^{-1} a_{M} \leqslant a_{M} a_{M}^{-1} \quad \text { and } \quad a_{M} h_{M}^{(1)} a_{M}=a_{M}^{2} .
$$

By induction

$$
h_{M}^{(i)}=a_{M}^{-i} a_{M}^{i} \quad \text { and } \quad a_{M} h_{M}^{(1)} a_{M} \cdots h_{M}^{(i)} a_{M}=a_{M}^{i+1},
$$

so for each $n \geqslant 1$,

$$
\overline{a^{n}}=a_{1} \cdots a_{M-1} a_{M}^{n}\left(h_{M+1}^{(n-1)} a_{M+1}\right) \cdots\left(h_{m}^{(n-1)} a_{m}\right)
$$

and since $a^{n}$ is an associate of $A, a_{1} \cdots a_{M-1} a_{M}^{n} h_{m+1}^{(n-1)} \in A$. But from the finiteness of $A$ it then follows that $a_{M}^{n}=a_{M}^{k}$ for some $n \neq k$. In that case $a_{M}^{-1} a_{M}=a_{M} a_{M}^{-1}$ and $a_{M}^{n-k}$ is idempotent. However, this contradicts the fact that $\overline{a^{n-k}}$ is reduced, completing the proof.

COROLlaRy 6.5. The free product of completely semisimple inverse semigroups is again completely semisimple.

The following lemma is required in the next proposition.

Lemma 6.6. Suppose $(A, a) \in F$, with $|A| \neq 1, l(a)>1$ and $A \rho_{a}=A$. Then $m$ is odd and $a=a_{1} \cdots a_{M-1} a_{M} a_{M-1}^{-1} \cdots a_{1}^{-1}$, where $M=(m+1) / 2$. 
Proof. In the notation of Lemma 6.3 (and assuming $a \in A_{S}$, say)

$$
\overline{a^{2}}=a_{1} \cdots a_{m}\left(h_{1}^{(1)} a_{1}\right) \cdots\left(h_{m}^{(1)} a_{m}\right) .
$$

Now since $A \rho_{a}=A, h_{1}^{(1)}, a_{1} a_{1}^{-1}$ and $a_{1}^{-1} a_{1}$ all belong to $A_{S}$, whence $h_{1}^{(1)}=a_{1} a_{1}^{-1}=$ $a_{1}^{-1} a_{1}$. Similarly $h_{i}^{(1)}=a_{i} a_{i}^{-1}=a_{i}^{-1} a_{i}, 1<i \leqslant m$, and in fact $h_{i}^{(n)}=a_{i} a_{i}^{-1}$ for all $n$ and $i$. Thus for each $n, \overline{a^{(n)}}=a^{(n)}=a$ and the hypotheses of Lemma 6.3 are satisfied. So $m$ is odd and $a_{m-i+1} a_{i}$ is idempotent for $1 \leqslant i \leqslant M-1$. Moreover, since both $a_{1} a_{1}^{-1}$ and $a_{m}^{-1} a_{m} \in A, a_{1} a_{1}^{-1}=a_{m}^{-1} a_{m}$. Thus $a_{m}=a_{1}^{-1}$. Similarly $a_{2} a_{2}^{-1}$ and $a_{m-1}^{-1} a_{m-1} \in A \rho_{a a_{1}}=A \rho_{a_{1} \cdots a_{m-1}}$, so $a_{m-1}=a_{2}^{-1}$ and by induction $a_{m-i+1}=a_{i}^{-1}$, $1 \leqslant i \leqslant M-1$, that is, $a=a_{1} \cdots a_{M-1} a_{M} a_{M-1}^{-1} \cdots a_{1}^{-1}$.

Proposition 6.7. Let $(A, 1) \in F,|A| \neq 1$. Then there exists $B$ in $\mathcal{Y}$ such that $H_{(A, 1)} \cong H_{(B, 1)}$ and $(B, s) \in H_{(B, 1)}$ if and only if $s=1$ or $s$ belongs to a finite subgroup $G$ of $H_{e}, e \in B_{S} \cup B_{T}$. In that case $H_{(A, 1)} \cong G$.

Proof. Clearly it may be assumed that $\left|H_{(A, 1)}\right|>1$. Suppose $(A, a) \mathcal{H}(A, 1)$ for some $a$ with $l(a)>1$. By Lemma 6.6, $a=a_{1} \cdots a_{M-1} a_{M} a_{M-1}^{-1} \cdots a_{1}^{-1}$, where $M=$ $(m+1) / 2$. Put $B=A \rho_{a_{1} \ldots a_{M-1}} ;$ then $a_{M} a_{M}^{-1} \in B$ and

$$
B=\left(A \rho_{a_{1} \cdots a_{M} a_{M-1}^{-1} \cdots a_{1}^{-1}}\right) \rho_{a_{1} \cdots a_{M-1}}=A \rho_{a_{1} \cdots a_{M}}=B \rho_{a_{M}},
$$

that is, $\left(B, a_{M}\right) \in H_{(B, 1)}$, with $a_{M} \neq 1$.

So it remains to consider the situation where $(A, s) \mathcal{H}(A, 1)$ for some $s \in S$, say (putting $B=A$ in that case). Suppose $H_{(A, 1)}$ also contains $(A, t)$ for some $t \in T$. Then $(A, s t) \in H_{(A, 1)}$, which is impossible since $l(s t)$ is even. On the other hand if $H_{(A, 1)}$ contains $(A, a)$ for some $a$ with $l(a)>1$ then, as above, $a=a_{1}$ $\cdots a_{M-1} a_{M} a_{M-1}^{-1} \cdots a_{1}^{-1}$. By a similar argument $a_{1} \notin T$. So $a_{1} \in S$ and $(A, \overline{s a}) \mathcal{H}(A, 1)$. But $\overline{s a}$ clearly does not have the required form. Hence if $(A, a) \mathcal{H}(A, 1)$ either $a=1$ or $a \in S$, in which case $a \in H_{e}, e=s s^{-1} \in A_{S}$. The map which takes $(A, a)$ to $a$, for $a \neq 1$, and $(A, 1)$ to $e$ is clearly an isomorphism of $H_{(A, 1)}$ upon a finite subgroup $G$, say, of $H_{e}$.

THEOREM 6.8. Every subgroup of $S$ inv $T$ which is not contained in $S$ or $T$ is isomorphic with a finite subgroup of $S$ or $T$. Conversely, every finite subgroup of $S$ or $T$ is isomorphic with a maximal subgroup of $S$ inv $T$.

Proof. Only the second statement remains to be proved. So let $e \in E(S)$, say, and suppose $G$ is a finite nontrivial subgroup of $H_{e}$. Let $f$ be an arbitrary idempotent of $T$.

Put $A=\{e\} \cup\{g f: g \in G, g \neq e\} \cup\{f\}$. It is clear that $A \in \mathcal{Y}$ and $(A, h) \mathcal{H}(A, 1)$ for all $h \in G$ (using the fact that left translation by $h^{-1}$ is a permutation of $G)$. Further, the map $h \rightarrow(A, h)$ is a monomorphism. As in the proof of the proposition, if $(A, a) \in H_{(A, 1)}, a \neq 1$, then $a \mathcal{H} e$. But $a^{-1} f \in A \rho_{a}=A$, so $a^{-1} \in G$. Hence $G \cong H_{(A, 1)}$.

COROLlARY 6.9. The free product of combinatorial inverse semigroups is again combinatorial. 
Next it will be shown that if neither $S$ nor $T$ has a zero, $S$ inv $T$ is fundamental. Recall that an inverse semigroup is fundamental $[3, \S 5.4]$ if $\mu$ is the identical relation. Unlike the $E$-unitary case [7, Theorem 5.4] there appear to be no simple necessary and sufficient conditions for fundamentality. For instance, in the $E$-unitary case if $S$ and $T$ are fundamental so is $S$ inv $T$. In the next section an example will be given showing that this is not true in general.

THEOREM 6.10. Let $S$ and $T$ be inverse semigroups without zero. Then $S \operatorname{inv} T$ is fundamental.

Proof. Suppose $(A, a) \mu(A, 1)$ in $F, a \neq 1$. Using Proposition 6.7 it may be assumed, without loss of generality, that $a=s \in S$ and $s s^{-1}=s^{-1} s=e$, say. Observe that for any $C \in \mathcal{Y}, C \leqslant A$, with $g \in C, g \leqslant e$, then since $C=C \rho_{s}=C \rho_{g s}$, a word $b \in C_{T}$ if and only if ( $\left.g s\right) b \in C_{S}$.

Suppose $A_{T}$ is empty (so that no word in $A_{S}$ begins with $s$ ): then for any $f \in E(T), C=A \cup\{f\}<A$ but $s f \notin C_{S}$, contradicting the above. So $A_{T}$ is nonempty. Let $b=b_{1} \cdots b_{n}$ be an inextensible member of $A_{T}$, in the sense that $b$ is not a prefix of any other member of $A_{T}$. Suppose $b_{n}$ is not the minimum idempotent of the factor to which it belongs. Then $b_{n}>f$, say, and since $A$ is right reduced, $b^{\prime}=b_{1}$ $\cdots b_{n-1} f \notin A$. Replacing $b$ by $b^{\prime}$ yields $C \in \mathcal{Y}$, with $C<A$ (since $\varepsilon(C)<\varepsilon(A)$ ) but $C \rho_{s} \neq C$, since $s b^{\prime} \notin C_{S}=A_{S}$. On the other hand, if $b_{n}$ is the minimum idempotent of the factor to which it belongs then since $b_{n}$ is not a zero, there is a nonidempotent $v \mathcal{H} b_{n}$. Let $g$ be any idempotent of the alternate factor and put $c=b_{1} \cdots b_{n-1} v f$. Since $b$ is inextensible, $c \notin A$. Thus $C=A \cup\{c\}<A$, which is again a contradiction since $s c \notin C_{S}=A_{s}$.

Hence $\mu$ is the identical congruence and $F$, and therefore $S \operatorname{inv} T$, is fundamental.

COROLlaRY 6.11. The free product of an infinite family of inverse semigroups is always fundamental.

Proof. This follows from the theorem and the observation that the free product of any infinite family of inverse semigroups cannot have a zero (since such an element can only involve finitely many factors).

7. Preservation properties of $S$ inv $T$. It is clear from the nature of the reduction process given in $\$ 3$ that if $S$ and $T$ have a soluble word problem then so does $S$ inv $T$.

In the previous section it was shown that the properties of complete semisimplicity and of being combinatorial are preserved by free products. In [8] it was shown (without using an explicit structure theorem) that the free product of $E$-unitary inverse semigroups is again $E$-unitary. (An inverse semigroup is $E$-unitary if $e a=e$, $e \in E(S), a \in S$ implies $a \in E(S)$, or equivalently, if $a \geqslant e$ implies $a \in E(S)$.) As an example of an application of Theorem 6.1, this will be deduced here.

Proposition 7.1. Let $S$ and $T$ be E-unitary inverse semigroups. Then $S \operatorname{inv} T$ is E-unitary. 
Proof. Suppose $(A, a) \geqslant(B, 1)$ in $F$. Then by Theorem 6.1(v), $\bar{a}_{B}=1$. If $a \neq 1$, let $g \in B, g_{i} \in B \rho_{a_{1} \cdots a_{i-1}}, 1<i \leqslant m$, be such that $g_{i} \leqslant a_{i} a_{i}^{-1}, 1<i \leqslant m$. Then $\bar{a}_{B}=\left(g_{1} a_{1}\right) \cdots\left(g_{m} a_{m}\right)$. But from the $E$-unitariness of $S$ and $T, \bar{a}_{B}$ is reduced as written. Hence $a=1$ and so $F$, and therefore $S$ inv $T$, is $E$-unitary.

O'Carroll [9] introduced the weaker notion of strong E-reflexivity: $S$ is strongly $E$-reflexive if exy $\in E(S), e \in E(S)$, implies eyx $\in E(S)$. He showed that strongly $E$-reflexive inverse semigroups are precisely the semilattices of $E$-unitary inverse semigroups.

Proposition 7.2. Let $S$ and $T$ be strongly E-reflexive inverse semigroups. Then $S$ inv $T$ is strongly E-reflexive. In particular, the free product of semilattices of groups is strongly E-reflexive.

Proof. Suppose $S[T]$ is a semilattice $Y_{S}\left[Y_{T}\right]$ of $E$-unitary inverse subsemigroups $S_{\alpha}, \alpha \in Y_{S}\left[T_{\beta}, \beta \in Y_{T}\right]$. Now $Y=Y_{S}$ inv $Y_{T}$ is a semilattice, in fact the free semilattice product of $Y_{S}$ and $Y_{T}$; thus each of its elements has the unique form $\alpha, \beta$ or $\alpha \beta$, where $\alpha \in Y_{S}, \beta \in Y_{T}$. From the universal properties of $S$ inv $T$ it is apparent that $S$ inv $T$ is then the semilattice $Y$ of inverse subsemigroups $F_{\gamma}, \gamma \in Y$, where $F_{\gamma}=S_{\gamma}$ if $\gamma \in Y_{S}, F_{\gamma}=T_{\gamma}$ if $\gamma \in Y_{T}$ and $F_{\gamma}$ is the inverse subsemigroup of $S$ inv $T$ generated by $S_{\alpha}$ and $T_{\beta}$ if $\gamma=\alpha \beta, \alpha \in Y_{S}, \beta \in Y_{T}$. Now, by Corollary 5.7, in this last case $F_{\gamma} \cong S_{\alpha}$ inv $T_{\beta}$, whence $F_{\gamma}$ is $E$-unitary.

Thus $S \operatorname{inv} T$ is a semilattice of $E$-unitary inverse semigroups, that is, $S \operatorname{inv} T$ is strongly $E$-reflexive.

In [6] the author discussed preservation of the Hopf property ("every onto endomorphism is an automorphism") under free products. In general the property is not preserved, but under a range of weak conditions it is.

In [4] the author introduced the strong basis property: an inverse semigroup $S$ has this property if for any inverse subsemigroups $U \subseteq V$ of $S$ any two $U$-bases for $V$ have the same cardinality, (a $U$-basis for $V$ being a set $X$ minimal with the property that $X \cup U$ generates $V$ ). It was shown there that free inverse semigroups have this property, from which it might be surmised that it is preserved by free products. However, as the following example shows, this is not the case. The example makes use of the characterization of all inverse semigroups with the strong basis property given in [5], to which the reader is referred for details.

EXAMPLE 7.3. Let $G$ be the symmetric group on three letters and let $H$ be a two element group. From Example 5.5 and Lemma 4.2 of [5] both $G$ and $H$ have the strong basis property. Let $e$ and $f$ denote the identities of $G$ and $H$, respectively, and let $H=\{f, h\}$. Put

$$
A=\{e\} \cup\{g f: g \in G, g \neq e\} \cup\{g h e: g \in G, g \neq e\} \cup\{h e, f\} .
$$

Then $A \in \mathcal{Y}=\mathcal{Y}(G, H)$ and $H_{(A, 1)} \cong G$, using an argument similar to that for Theorem 6.8. Further $H_{(A, 1)}$ is nonisolated, that is, $H_{(A, 1)} \neq D_{(A, 1)}$ (since $(A, g h) \in F$ but $A \rho_{g h} \neq A$ since $l(g h)=2$ ). But by [4, Theorem 4.8] any nonisolated maximal subgroup of an inverse semigroup with the strong basis property must be primary (that is, a $p$-group for some prime $p$ ). Clearly $G$ is not primary. 

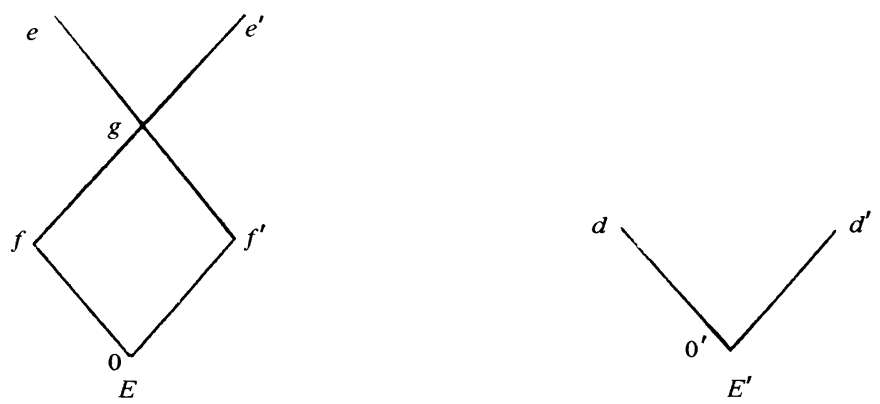

\section{Figure 1}

Finally an example is given of two fundamental inverse semigroups whose free product is not fundamental.

EXAmple 7.4. Let $E$ and $E^{\prime}$ be the semilattices in Figure 1, and let $S=T_{E}$, $T=T_{E^{\prime}}$, the Munn semigroups on $E$ and $E^{\prime}$ respectively; thus $S$ and $T$ are fundamental. In each case the idempotents (as identified in the figure) will be identified with the identical automorphisms of the principal ideals they generate.

Clearly $T=\left\{0^{\prime}, d, d^{\prime}, t, t^{-1}\right\}$, where $t$ is the isomorphism of $E^{\prime} d$ upon $E^{\prime} d^{\prime}$ : thus $T$ is isomorphic with the five element combinatorial Brandt semigroup $B_{5}$.

Denote by $s$ the automorphism of $E e$ which interchanges $f$ and $f^{\prime}$, and by $a$ the isomorphism of $E e$ upon $E e^{\prime}$ which leaves $E g$ fixed. Then $s a=b$, say, is the isomorphism of $E e$ upon $E e^{\prime}$ which interchanges $f$ and $f^{\prime}$. Note that $g s=g b$ and $g a=g e=g$; and that $f s=f b$ is the isomorphism of $E f$ upon $E f^{\prime}$. Hence $S=D_{e} \cup$ $D_{g} \cup D_{f} \cup D_{0}$, where $D_{e}$ consists of the four $\mathcal{H}$-classes $\{e, s\},\{a, b\}$, $\left\{a^{-1}, b^{-1}\right\},\left\{e^{\prime}, a^{-1} b\right\}, D_{g}$ is the group $\{g, g s\}$, and $D_{f} \cup\{0\}=\left\{f, f s, f^{\prime}, s^{-1} f, 0\right\}$ is again isomorphic with $B_{5}$.

Now put $A=\left\{e, s 0^{\prime}, a d, b d\right.$, at $\left.0, g t 0\right\} \cup\left\{0^{\prime}\right\}$. It is clear that $A \in \mathcal{O}$ and (since $s=s^{-1}$ and $\left.s a=b, s b=a\right) A \rho_{s}=A$. It will now be shown that $A$ covers the zero $\left\{0,0^{\prime}\right\}$ of $\mathcal{Y}$, from which it follows that $(A, s) \mu(A, 1)$ and $F$ is not fundamental.

So let $B \in \mathcal{Y}, B \leqslant A$. Since $0^{\prime}$ is the zero of $T$ it is clear that $B_{T}=\left\{0^{\prime}\right\}$. Suppose $e \notin B$ : then $e>h$ for some $h \in B$, and so $A \wedge\{g\} \geqslant B$. Thus, in $S \operatorname{inv} T$,

$$
\begin{aligned}
\varepsilon(B) & \leqslant \varepsilon(A) \varepsilon(g)=\left(s 0^{\prime} s^{-1}\right)\left(a t 0 t^{-1} a^{-1}\right)\left(b t 0 t^{-1} b^{-1}\right) 0^{\prime} g \\
& =\left((g s) 0^{\prime}(g s)^{-1}\right)\left(t 0 t^{-1}\right)\left((g b) t 0 t^{-1}(g b)^{-1}\right) 0^{\prime}, \quad \text { since } g a=g, \\
& =00^{\prime}=\varepsilon\left(\left\{0,0^{\prime}\right\}\right), \quad \text { since } 0^{\prime} t=0^{\prime},
\end{aligned}
$$

whence $B=\left\{0,0^{\prime}\right\}$. (A direct calculation of $A \wedge\{g\}=\operatorname{cl}(A \cup g)$ is also possible.)

On the other hand, if $e \in B$ then $s 0^{\prime} \in B$ for, by Proposition 4.10, $s 0^{\prime}$ is above a pseudomember $b_{1} b_{2}$, say, of $B$, in which case $b_{2}=0^{\prime}$ and, since $b_{1} \leqslant s$ and $b_{1} R e$, $b_{1}=s$. Similarly, either $a d \in B$ or $a 0^{\prime} \in B$. In the latter case $a 0^{\prime} 0$ must be the pseudomember of $B$ below the member at 0 of $A$, in which case $0^{\prime} 0=\varepsilon\left(0^{\prime} 0\right) \geqslant \varepsilon\left(B \rho_{a}\right)$, so $B \rho_{a}=\left\{0,0^{\prime}\right\}$ and $B=\left\{0,0^{\prime}\right\}$ itself.

Similarly, either $B=\left\{0,0^{\prime}\right\}$ or at $0, b d$ and bt 0 belong to $B$, that is, $A \subseteq B$. But since $R_{e}=\{e, s, a, b\}$ and $R_{d}=\{d, t\}$, in that case $A=B$, completing the argument. 


\section{REFERENCES}

1. G. Grätzer, Universal algebra, Springer-Verlag, New York, 1979.

2. T. E. Hall, Inverse and regular semigroups and amalgamation: a brief survev, Proc. Sympos. Regular Semigroups, Northern Illinois Univ., DeKalb, Ill., 1979, pp. 49-79.

3. J. M. Howie, An introduction to semigroup theory, Academic Press, London, 1976.

4. P. R. Jones, A basis theorem for free inverse semigroups, J. Algebra 49 (1977), 172-190.

5. __ Basis properties for inverse semigroups, J. Algebra 50 (1978), 135-152.

6. The Hopf property and K-free products of semigroups, Semigroup Forum 20 (1980), $343-368$.

7. A graphical representation for the free product of E-unitary inverse semigroups, Semigroup Forum 24 (1982), 195-221.

8. N. Knox, On the inverse semigroup coproduct of an arbitrary nonempty collection of groups, Ph.D. Thesis, Tennessee State Univ., Nashville, Tennessee, 1974.

9. D. B. McAlister, Inverse semigroups generated by a pair of subgroups, Proc. Roy. Soc. Edinburgh Sect. A 77 (1976), 9-22.

10. L. O'Carroll, Strongly E-reflexive inverse semigroups, Proc. Edinburgh Math. Soc. (2) 20 (1976-7), $339-354$.

11. G. B. Preston, Inverse semigroups: some open questions, Proc. Sympos. Inverse Semigroups and Their Generalizations, Northern Illinois Univ., DeKalb, Ill., 1973, pp. 122-139.

12. H. E. Scheiblich, Free inverse semigroups, Proc. Amer. Math. Soc. 38 (1973), 1-7.

Department of Mathematics, Statistics and Computer Science, Marquette University, MilWAUKEe, Wisconsin 53233 